\title{
Dislocations in an anisotropic Swift-Hohenberg equation
}

\author{
Mariana Haragus ${ }^{a}$ \& Arnd Scheel ${ }^{b}$ \\ ${ }^{a}$ Université de Franche-Comté, Laboratoire de Mathématiques, 16 route de Gray, 25030 Besançon cedex, France \\ ${ }^{b}$ University of Minnesota, School of Mathematics, 206 Church St. S.E., Minneapolis, MN 55455, USA
}

May 3, 2012

\begin{abstract}
We study the existence of dislocations in an anisotropic Swift-Hohenberg equation. We find dislocations as traveling or standing waves connecting roll patterns with different wavenumbers in an infinite strip. The proof is based on a bifurcation analysis. Spatial dynamics and center-manifold reduction yield a reduced, coupled-mode system of differential equations. Existence of traveling dislocations is then established by showing that this reduced system possesses robust heteroclinic orbits.
\end{abstract}

Running head: Dislocations in a modified Swift-Hohenberg equation

Corresponding author: Mariana Haragus, mharagus@univ-fcomte.fr

Keywords: Swift-Hohenberg equation, roll solution, dislocation 


\section{Introduction}

Defects in patterns occur with a striking universality and regularity in a wide range of systems. The arguably best-studied experimental setup is the Rayleigh-Bénard convection experiment, where convection roll patterns with a variety of embedded defects form close to onset. Defects in crystal patterns also play a fundamental role in material science. In both scenarios, the Swift-Hohenberg equation, which in its simplest form reads

$$
u_{t}=-(\Delta+1)^{2} u+\mu u-u^{3},
$$

on $\mathbb{R}^{n}, n=1,2,3$, has been used as a prototypical model system that mimics phenomena qualitatively (and sometimes even quantitatively), while being analytically and numerically easily tractable; see for instance [2]. The crucial property of the Swift-Hohenberg equation is the instability of a spatially homogeneous state with respect to roll patterns $u(X) \sim \cos (K \cdot X)$, with $X \in \mathbb{R}^{n}$ and wavenumber $K \in \mathbb{R}^{n},|K| \sim 1$, for parameter values $\mu$ above criticality. Depending on the nonlinearity, this leads to the creation of stable nonlinear patterns that resemble these linear modes. We are interested in situations where nonlinear roll patterns bifurcate in a stable fashion. Our aim is to prove the existence of dislocations in planar spatially extended systems. In this context, dislocations are stationary solutions in an appropriate frame of reference, that resemble roll patterns $\cos (K \cdot X+2 \pi \varphi(X))$ with constant orientation $K \sim(0,1)^{T} \in \mathbb{R}^{2}$ and appropriate phase shift function $\varphi(X)$ in the far field. Associated with such patterns is a topological charge, obtained by integrating the phase $\varphi$ along a circle in the far field. For dislocations, this topological charge is \pm 1 : the number of rolls along the vertical line $(x, y) \sim(-R, y), R \gg 1$, differs from the number along the line $(x, y) \sim(+R, y)$ by one; see for instance $[2,9]$ for some background and references.

Despite the variational structure of the problem, proving existence of such solutions appears to be a quite delicate problem. Most attempts have focused on the analysis of phase modulation equations; see for instance $[3,4,5]$. Our approach here is slightly different in spirit. We will find dislocations as solutions in a strip $(x, y) \in \mathbb{R} \times \mathbb{R} / L \mathbb{Z}$, with $L$-periodic boundary conditions in $y$; see Figure 1.1 for a schematic plot. Such boundary conditions accommodate roll solutions of the form $u \sim \cos (k y)$ when $k$ solves $j \cdot \frac{2 \pi}{k}=L$ for some $j \in \mathbb{Z}$. When $L$ is large, this allows for wavenumbers $k_{-}:=j \cdot \frac{2 \pi}{L} \lesssim 1 \lesssim$ $(j+1) \cdot \frac{2 \pi}{L}=: k_{+}$. The corresponding roll solutions bifurcate from the trivial solutions for $\mu \sim 0$ and we can, in principle, attempt to find traveling waves (or interfaces) between these rolls with wavenumbers $k_{-}, k_{+}$. By construction, the winding number defined above is indeed \pm 1 , when evaluated on $|x|=R$, $0 \leqslant y \leqslant L$. Extended to solutions in the plane $(x, y) \in \mathbb{R} \times \mathbb{R}$, such solutions represent a periodic alignment of dislocations in the $y$-direction.

It turns out that our approach reveals an obstacle to the construction of such standing (or slow moving) dislocations in the form of resonances. In fact, together with rolls of the form $\cos (k y), k \sim 1$, we have

rolls of the form $\cos \left(k_{x} x+k_{y} y\right)$, when $k_{x}^{2}+k_{y}^{2} \sim 1$. With the above restriction $k_{y} \in \frac{2 \pi}{L} \mathbb{Z}$, this allows for a plethora of roll solutions compatible with the boundary conditions when $L$ is chosen large enough. Our present approach avoids this difficulty by considering an anisotropic version of the Swift-Hohenberg equation

$$
\partial_{t} u=-(1+\Delta)^{2} u+\mu u+\beta \partial_{x}^{2} u-u^{3} .
$$

Here, $u$ depends upon the two spatial variables $(x, y) \in \mathbb{R}^{2}$ and time $t \in \mathbb{R}, \mu$ is a small real parameter, 

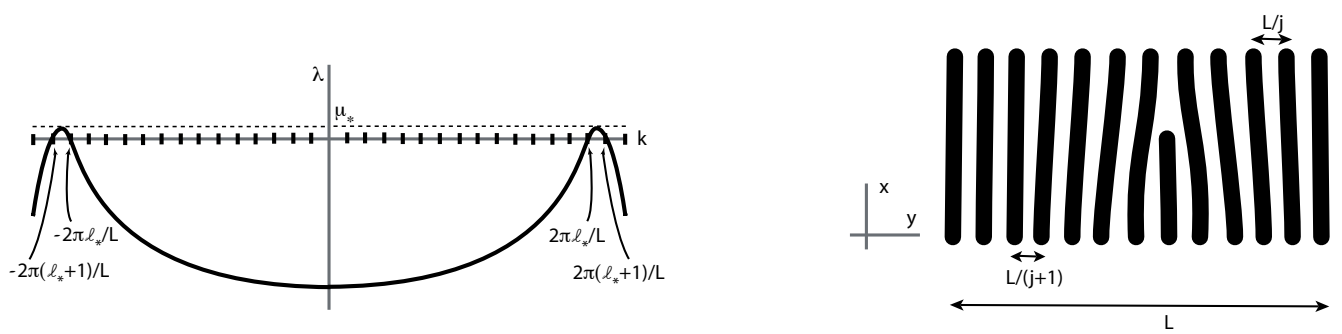

Figure 1.1: On the left, a schematic plot of the linear dispersion curve of the Swift-Hohenberg equation, $\lambda=$ $-\left(1-k^{2}\right)^{2}+\mu$, quantized in steps $2 \pi j / L$ due to periodicity in $y$. Only the modes $j= \pm \ell_{*}$ and $j= \pm\left(\ell_{*}+1\right)$ contribute to the kernel at parameter value $\mu=\mu_{*}$. On the right, a schematic picture of a dislocation with horizontal $y$-periodicity.

and $\beta>0$. The effect of $\beta$ is an effective linear damping of modes with wavenumber $k_{x}>0$, so that only horizontal rolls, $k_{x}=0$, exist for $\mu \sim 0$.

While the damping of vertical modes effectively simplifies the problem, we believe that the resulting bifurcation problem here is related to the problem in the isotropic case. One would expect that the coupling between rolls of different orientation is weak in the isotropic case, a fact that can be made precise using reduction methods and normal form theory similar to the present paper. As a result, we would expect that the existence problem in the isotropic case reduces to an interaction between two roll-modes similarly to the present anisotropic case. On the other hand, the isotropic case creates an additional complication due to transverse zig-zag instabilities, which might effectively prevent existence of certain dislocations; see also the discussion in Section 5.4 and Figure 5.1.

Beyond mimicking some of the features of the isotropic Swift-Hohenberg equation, the anisotropic SwiftHohenberg equation itself plays an important role in modeling anisotropic convection [11], nematic liquid crystals $[1,7]$, or, more generally, anisotropic pattern-forming systems. The effect of anisotropy on point defects has been studied in [10]. The results show that the anisotropy favors dislocations against other point defects such as disclinations.

We construct the dislocations as traveling-wave solutions to the equation (1.1), which propagate in the direction $x$ with speed $c$, and which are periodic in $y$ with period $L=2 \pi / k$. Setting $\tilde{x}:=x+c t$, rescaling $k y=: \tilde{y}$, and dropping tildes, we find the traveling-wave equation

$$
c \partial_{x} u=-\left(1+\partial_{x}^{2}+k^{2} \partial_{y}^{2}\right)^{2} u+\mu u+\beta \partial_{x}^{2} u-u^{3},
$$

with $2 \pi$-periodic boundary conditions in $y$. A dislocation is a solution of (1.2), which is asymptotic, as $x \rightarrow-\infty$ and $x \rightarrow+\infty$, to two $x$-independent solutions, with different periods $\frac{2 \pi}{\ell_{-}}$and $\frac{2 \pi}{\ell_{+}}$, respectively. Our main result restricts to the simplest case when $\left|\ell_{-}-\ell_{+}\right|=1$.

The construction of dislocations is carried out for parameters $\mu$ close to zero, while $\beta>0$ is fixed. The parameter $c$ is taken as a free parameter close to zero. More specifically, we consider parameter values $\mu=\mu_{*}+\bar{\mu}$, where $\mu_{*}$ is the parameter value for which two roll solutions with common minimal $y$-period $L \gg 1$ bifurcate from the origin. The amplitude of these roll solutions is, as usual, $\mathrm{O}\left(\bar{\mu}^{1 / 2}\right)$.

Theorem 1 (Existence of dislocations) Consider $\beta>0, k_{*}>0$ and $\mu_{*}>0$ defined by

$$
k_{*}^{2}=\frac{2}{2 \ell_{*}^{2}+2 \ell_{*}+1}, \quad \sqrt{\mu_{*}}=\frac{2 \ell_{*}+1}{2 \ell_{*}^{2}+2 \ell_{*}+1},
$$


for $\ell_{*} \in \mathbb{N}$, and set

$$
\mu=\mu_{*}+\bar{\mu}
$$

For any $\ell_{*}$ sufficiently large, and any $\bar{\mu}$ such that $\bar{\mu} \mu_{*}^{-2}$ is sufficiently small, the modified SwiftHohenberg equation (1.1) possesses a traveling wave

$$
\begin{aligned}
u(x, y, t)= & \frac{2}{\sqrt{3}} \bar{\mu}^{1 / 2} A_{0}^{\star}\left(\frac{1}{\sqrt{\beta}} \bar{\mu}^{1 / 2}(x+c t)\right) \cos \left(k_{*} \ell_{*} y\right) \\
& +\frac{2}{\sqrt{3}} \bar{\mu}^{1 / 2} B_{0}^{\star}\left(\frac{1}{\sqrt{\beta}} \bar{\mu}^{1 / 2}(x+c t)\right) \cos \left(k_{*}\left(\ell_{*}+1\right) y\right)+\mathcal{O}\left(\bar{\mu}^{1 / 2} \mu_{*}^{1 / 2}+\bar{\mu} \mu_{*}^{-1}\right),
\end{aligned}
$$

with speed $c=\mathcal{O}\left(\bar{\mu}^{1 / 2} \mu_{*}^{1 / 2}+\bar{\mu} \mu_{*}^{-1}\right)$, which is $\frac{2 \pi}{k_{*}}$-periodic in $y$. Here $\left(A_{0}^{\star}, B_{0}^{\star}\right)$ is the heteroclinic orbit in Lemma 4.1, so that in particular

$$
\lim _{x \rightarrow-\infty} u(x, y, t)=\frac{2}{\sqrt{3}} \bar{\mu}^{1 / 2} \cos \left(k_{*} \ell_{*} y\right), \quad \lim _{x \rightarrow \infty} u(x, y, t)=\frac{2}{\sqrt{3}} \bar{\mu}^{1 / 2} \cos \left(k_{*}\left(\ell_{*}+1\right) y\right) .
$$

Outline. We formulate the traveling-wave problem as an ill-posed dynamical system and analyze spectra of the linearization in Section 2. In particular, we determine parameter values $\mu_{*}$ where roll solutions with different winding number $\ell_{ \pm}$bifurcate from the origin. Section 3 contains the main reduction result, including estimates on the resolvent and nonlinear parts in suitable scalings. Section 4 is concerned with an analysis of the reduced equations, showing existence, robustness, and various qualitative properties of the heteroclinic orbit. We briefly illustrate our results in Section 5 with numerical computations and compare direct simulations with predictions from theory. We also compare with results obtained from amplitude equations and with simple predictions from the variational structure, and discuss some generalizations.

Acknowledgments. This work was partially supported by the Agence Nationale de la Recherche through grant ANR PREFERED (M.H.) and the National Science Foundation through grant NSFDMS-0806614 (A.S.). The second author gratefully acknowledges support and generous hospitality by the Université de Franche-Comté, grant of the Franche-Comté region, during a visit in the fall of 2007 when much of the present work was carried out. The authors also would like to thank the anonymous referee for comments that helped to improve the paper significantly.

\section{Spatial dynamics and critical parameter values}

We write the equation (1.2) as a first order differential equation in $x$,

$$
U_{x}=\mathcal{A}(\mu, k, c, \beta) U+\mathcal{F}(U),
$$

where

$$
U=\left(\begin{array}{c}
u \\
u_{1} \\
v \\
v_{1}
\end{array}\right), \quad \mathcal{A}(\mu, k, c, \beta)=\left(\begin{array}{cccc}
0 & 1 & 0 & 0 \\
-\left(1+k^{2} \partial_{y}^{2}\right) & 0 & 1 & 0 \\
0 & 0 & 0 & 1 \\
-\beta\left(1+k^{2} \partial_{y}^{2}\right)+\mu & c & -\left(1+k^{2} \partial_{y}^{2}\right)+\beta & 0
\end{array}\right), \quad \mathcal{F}(U)=\left(\begin{array}{c}
0 \\
0 \\
0 \\
-u^{3}
\end{array}\right),
$$


with

$$
u_{1}=\partial_{x} u, \quad v=\left(1+\partial_{x}^{2}+k^{2} \partial_{y}^{2}\right) u, \quad v_{1}=\partial_{x} v .
$$

We view (2.1) as a dynamical system in the infinite-dimensional phase space

$$
\mathcal{X}=H_{\mathrm{per}}^{3}(0,2 \pi) \times H_{\mathrm{per}}^{2}(0,2 \pi) \times H_{\mathrm{per}}^{1}(0,2 \pi) \times L^{2}(0,2 \pi),
$$

consisting of $2 \pi$-periodic functions,

$$
H_{\mathrm{per}}^{j}(0,2 \pi)=\left\{u \in H_{\mathrm{loc}}^{j}(\mathbb{R}) ; u(z+2 \pi)=u(z), \text { for all } z \in \mathbb{R}\right\}, \quad j \geqslant 1 .
$$

Here $\mathcal{X}$ is equipped with the standard product norm for Hilbert spaces, and $H_{\text {per }}^{j}(0,2 \pi), j \geqslant 1$, with the norm

$$
\|u\|_{j}^{2}=\|u\|^{2}+\left\|k^{j} \partial_{y}^{j} u\right\|^{2}
$$

where $\|\cdot\|$ is the norm in $L^{2}(0,2 \pi)$,

$$
\|u\|^{2}=\int_{0}^{2 \pi}|u(z)|^{2} d z .
$$

Notice that in the original coordinates of equation (1.1), i.e., before the rescaling $k y=: \tilde{y}$, this norm of $H_{\text {per }}^{j}(0,2 \pi)$ gives rise to the usual $H^{j}$-norm of periodic functions. The linear part $\mathcal{A}(\mu, k, c, \beta)$ is a closed linear operator in $\mathcal{X}$, with dense domain of definition

$$
\mathcal{Y}=H_{\text {per }}^{4}(0,2 \pi) \times H_{\text {per }}^{3}(0,2 \pi) \times H_{\text {per }}^{2}(0,2 \pi) \times H_{\text {per }}^{1}(0,2 \pi),
$$

and the nonlinear map $\mathcal{F}: \mathcal{Y} \rightarrow \mathcal{Y}$ is smooth.

In this setting, solutions of equation (1.2) which are $x$-independent and $2 \pi$-periodic in $y$ correspond to equilibria of the dynamical system (2.1). Dislocations in turn correspond to heteroclinic orbits connecting two such nontrivial equilibria. We expect such heteroclinic orbits to bifurcate when two nontrivial equilibria bifurcate. Parameter values of $\mu, k$, and $c$ for such a steady-state bifurcation can be determined from the linearized equation

$$
U_{x}=\mathcal{A}(\mu, k, \beta, c) U
$$

which should possess two nontrivial solutions of the form $U(x, y)=e^{i \ell_{ \pm} y} U_{*}, U_{*} \in \mathbb{C}^{4}, \ell_{ \pm} \in \mathbb{Z}, \ell_{-} \neq \ell_{+}$. Rewriting the first-order equation as a higher-order equation, this is, of course, equivalent to a condition on the linearized traveling-wave equation

$$
c \partial_{x} u=-\left(1+\partial_{x}^{2}+k^{2} \partial_{y}^{2}\right)^{2} u+\mu u+\beta \partial_{x}^{2} u,
$$

which should possess two nontrivial solutions of the form $u(x, y)=e^{i \ell_{ \pm} y} u_{*}, u_{*} \in \mathbb{C}, \ell_{ \pm} \in \mathbb{Z}, \ell_{-} \neq \ell_{+}$.

We will need somewhat more general information on the linear equation (2.2). We therefore substitute the ansatz $U(x, y)=e^{\nu x+i \ell y} U_{*}$, with $\nu \in \mathbb{C}$ and $\ell \in \mathbb{Z}$, into the linear equation (2.2). We find a solution whenever $\nu$ is a root of the dispersion relation,

$$
c \nu=-\left(1+\nu^{2}-k^{2} \ell^{2}\right)^{2}+\mu+\beta \nu^{2} .
$$


In particular, the equation (2.2) possesses two nontrivial solutions of the form $u(x, y)=e^{i \ell_{ \pm} y} u_{*}, u_{*} \in \mathbb{C}$, $\ell_{-} \neq \ell_{+}$, if $\nu=0$ and $\ell=\ell_{ \pm}$are roots of the dispersion relation. This in turn can be interpreted as imposing two conditions on the parameters $\mu$ and $k$,

$$
\mu=\left(1-k^{2} \ell_{ \pm}^{2}\right)^{2}
$$

We note that this analysis is independent of the wave speed $c$ and the (fixed) parameter $\beta$. We will later set $c=0$ at criticality.

Restricting to the case $\left|\ell_{-}-\ell_{+}\right|=1$, we set $\ell_{-}=\ell_{*}, \ell_{+}=\ell_{*}+1, \ell_{*} \in \mathbb{N}$, so that the conditions $(2.3)$ now read

$$
\mu=\left(1-k^{2} \ell_{*}^{2}\right)^{2}, \quad \mu=\left(1-k^{2}\left(\ell_{*}+1\right)^{2}\right)^{2} .
$$

For a given $\ell_{*} \in \mathbb{N}$, these two equations have unique solutions

$$
\mu=\mu_{*}>0, \quad k=k_{*}>0 .
$$

In fact, we find

$$
k_{*}^{2} \ell_{*}^{2}=1-\sqrt{\mu_{*}}, \quad k_{*}^{2}\left(\ell_{*}+1\right)^{2}=1+\sqrt{\mu_{*}},
$$

which in turn gives the explicit values

$$
k_{*}^{2}=\frac{2}{2 \ell_{*}^{2}+2 \ell_{*}+1}, \quad \sqrt{\mu_{*}}=\frac{2 \ell_{*}+1}{2 \ell_{*}^{2}+2 \ell_{*}+1} .
$$

Remark 2.1 Later we will need to assume that $\mu_{*}$ is sufficiently small, which by the above is equivalent to assuming that $\ell_{*}$ is sufficiently large. In this regime, we have

$$
k_{*}^{2}=\frac{1}{\ell_{*}^{2}}+\mathcal{O}\left(\frac{1}{\ell_{*}^{3}}\right), \quad \sqrt{\mu_{*}}=\frac{1}{\ell_{*}}+\mathcal{O}\left(\frac{1}{\ell_{*}^{2}}\right) .
$$

In the subsequent analysis, we therefore keep track of the dependence of various quantities on $\ell_{*}$, in the limit $\ell_{*} \rightarrow \infty$. We use the sub- or super-script $*$ to indicate such quantities that depend on the choice of $\ell_{*}\left(\right.$ or $\left.\mu_{*}\right)$.

\section{Linear estimates, scaling, and reduction}

We choose $\ell_{*}$ large, to be determined later, and $k_{*}, \mu_{*}$ as in (2.5). We fix the parameters $k=k_{*}$ and $\beta>0$, and take $\mu \sim \mu_{*}$ and $c \sim 0$ as bifurcation parameters. We set

$$
\mu=\mu_{*}+\bar{\mu}, \quad k=k_{*} .
$$

Making explicit the dependence of the linear part on parameters, we write the system (2.1) in the form

$$
U_{x}=\mathcal{A}_{*} U+\mathcal{B}(\bar{\mu}, c) U+\mathcal{F}(U)
$$

in which

$$
\mathcal{A}_{*}=\mathcal{A}\left(\mu_{*}, k_{*}, 0, \beta\right), \quad \mathcal{B}(\bar{\mu}, c)=\mathcal{A}\left(\mu_{*}+\bar{\mu}, k_{*}, c, \beta\right)-\mathcal{A}\left(\mu_{*}, k_{*}, 0, \beta\right) .
$$


The linear operator. The principal part of the linearization $\mathcal{A}_{*}$ is closed in $\mathcal{X}$, with dense and compactly embedded domain $\mathcal{Y}$. In particular, $\mathcal{A}_{*}$ has compact resolvent and its spectrum is pure point spectrum, only. The eigenvalues of $\mathcal{A}_{*}$ are determined by the dispersion relation,

$$
\sigma\left(\mathcal{A}_{*}\right)=\left\{\nu \in \mathbb{C} ;-\left(1+\nu^{2}-k_{*}^{2} \ell^{2}\right)^{2}+\mu_{*}+\beta \nu^{2}=0, \ell \in \mathbb{Z}\right\} .
$$

One can easily see that imaginary eigenvalues, $\nu \in \mathbb{i}$, yield eigenfunctions to the PDE linearization of the form $\mathrm{e}^{\nu x+\mathrm{i} k_{*} \ell y}$. The anisotropic damping $\beta \partial_{x x}$ suppresses such modes with $0 \neq \nu \in \mathrm{i} \mathbb{R}$. In fact, a direct calculation shows that

$$
\sigma\left(\mathcal{A}_{*}\right) \cap \mathrm{i} \mathbb{R}=\{0\},
$$

if we assume sufficiently strong anisotropy or $\mu_{*}$ sufficiently close to criticality, i.e., if

$$
\beta-2 \sqrt{\mu_{*}}>0
$$

Moreover, one finds that 0 is an eigenvalue with geometric multiplicity four and algebraic multiplicity eight, and that

$$
\sigma\left(\mathcal{A}_{*}\right) \backslash\{0\} \subset\left\{\lambda \in \mathbb{C} ;|\operatorname{Re} \lambda| \geqslant \delta_{*}\right\},
$$

for some $\delta_{*}>0$, with $\delta_{*}=\mathcal{O}\left(\sqrt{\mu_{*}}\right)$, as $\mu_{*} \rightarrow 0$. The eigenvectors associated with the eigenvalue 0 correspond to the Fourier modes $\ell_{*}$ and $\ell_{*}+1$, and are given by

$$
E_{ \pm \ell_{*}}(y)=\left(\begin{array}{c}
1 \\
0 \\
\sqrt{\mu_{*}} \\
0
\end{array}\right) e^{ \pm \mathrm{i} \ell_{*} y}, \quad E_{ \pm\left(\ell_{*}+1\right)}(y)=\left(\begin{array}{c}
1 \\
0 \\
-\sqrt{\mu_{*}} \\
0
\end{array}\right) e^{ \pm \mathrm{i}\left(\ell_{*}+1\right) y}
$$

Each of these eigenvectors leads to a Jordan chain of length 2, with principal eigenvectors

$$
F_{ \pm \ell_{*}}(y)=\left(\begin{array}{c}
0 \\
1 \\
0 \\
\sqrt{\mu_{*}}
\end{array}\right) e^{ \pm \mathrm{i} \ell_{*} y}, \quad F_{ \pm\left(\ell_{*}+1\right)}(y)=\left(\begin{array}{c}
0 \\
1 \\
0 \\
-\sqrt{\mu_{*}}
\end{array}\right) e^{ \pm \mathrm{i}\left(\ell_{*}+1\right) y}
$$

satisfying

$$
\mathcal{A}_{*} F_{ \pm \ell_{*}+1}(y)=E_{ \pm \ell_{*}}(y), \quad \mathcal{A}_{*} F_{ \pm\left(\ell_{*}+1\right)}(y)=E_{ \pm\left(\ell_{*}+1\right)}(y) .
$$

These eigenvectors span the eight-dimensional spectral subspace $\mathcal{Y}_{*}$ of $\mathcal{A}_{*}$ associated to the eigenvalue 0 . We also need to compute the spectral projection $\mathcal{P}_{*}: \mathcal{X} \rightarrow \mathcal{Y}_{*}$ onto this spectral subspace. Denoting by $\langle\cdot, \cdot\rangle$ the scalar product in $\left(L^{2}(0,2 \pi)\right)^{4}$, the spectral projection is given by

$$
\mathcal{P}_{*} U=\sum_{\kappa \in\left\{ \pm \ell_{*}, \pm\left(\ell_{*}+1\right)\right\}}\left(\left\langle U, E_{\kappa}^{\mathrm{ad}}\right\rangle E_{\kappa}+\left\langle U, F_{\kappa}^{\mathrm{ad}}\right\rangle F_{\kappa}\right),
$$

where

$$
\left\langle E_{\kappa}, E_{\kappa}^{\mathrm{ad}}\right\rangle=\left\langle F_{\kappa}, F_{\kappa}^{\mathrm{ad}}\right\rangle=1, \quad\left\langle F_{\kappa}, E_{\kappa}^{\mathrm{ad}}\right\rangle=\left\langle E_{\kappa}, F_{\kappa}^{\mathrm{ad}}\right\rangle=0, \quad \mathcal{A}_{*}^{\mathrm{ad}} F_{\kappa}^{\mathrm{ad}}=0, \quad \mathcal{A}_{*}^{\mathrm{ad}} E_{\kappa}^{\mathrm{ad}}=F_{\kappa}^{\mathrm{ad}},
$$


and $\mathcal{A}_{*}^{\text {ad }}$ is the adjoint of $\mathcal{A}_{*}$ with respect to this scalar product,

$$
\mathcal{A}_{*}^{\mathrm{ad}}=\left(\begin{array}{cccc}
0 & -\left(1+k_{*}^{2} \partial_{y}^{2}\right) & 0 & -\beta\left(1+k_{*}^{2} \partial_{y}^{2}\right)+\mu_{*} \\
1 & 0 & 0 & 0 \\
0 & 1 & 0 & -\left(1+k_{*}^{2} \partial_{y}^{2}\right)+\beta \\
0 & 0 & 1 & 0
\end{array}\right) .
$$

A direct calculation gives

$$
\begin{aligned}
& F_{ \pm \ell_{*}}^{\mathrm{ad}}(y)=\frac{1}{2 \pi\left(\beta-2 \sqrt{\mu_{*}}\right)}\left(\begin{array}{c}
0 \\
\beta-\sqrt{\mu_{*}} \\
0 \\
-1
\end{array}\right) e^{ \pm \mathrm{i} \ell_{*} y}, \quad F_{ \pm\left(\ell_{*}+1\right)}^{\mathrm{ad}}(y)=\frac{1}{2 \pi\left(\beta+2 \sqrt{\mu_{*}}\right)}\left(\begin{array}{c}
0 \\
\beta+\sqrt{\mu_{*}} \\
0 \\
-1
\end{array}\right) e^{ \pm \mathrm{i}\left(\ell_{*}+1\right) y}, \\
& E_{ \pm \ell_{*}}^{\mathrm{ad}}(y)=\frac{1}{2 \pi\left(\beta-2 \sqrt{\mu_{*}}\right)}\left(\begin{array}{c}
\beta-\sqrt{\mu_{*}} \\
0 \\
-1 \\
0
\end{array}\right) e^{ \pm \mathrm{i} \ell_{*} y}, \quad E_{ \pm\left(\ell_{*}+1\right)}^{\mathrm{ad}}(y)=\frac{1}{2 \pi\left(\beta+2 \sqrt{\mu_{*}}\right)}\left(\begin{array}{c}
\beta+\sqrt{\mu_{*}} \\
0 \\
-1 \\
0
\end{array}\right) e^{ \pm \mathrm{i}\left(\ell_{*}+1\right) y} .
\end{aligned}
$$

As $\mu_{*} \rightarrow 0$, the norms in $\mathcal{X}$ and $\mathcal{Y}$ of all these vectors is $\mathcal{O}(1)$ which implies that we have uniform estimates for the spectral projection $\mathcal{P}_{*}$,

$$
\left\|\mathcal{P}_{*}\right\|_{\mathcal{L}(\mathcal{X}, \mathcal{X})}=\mathcal{O}(1), \quad\left\|\mathcal{P}_{*}\right\|_{\mathcal{L}(\mathcal{Y}, \mathcal{Y})}=\mathcal{O}(1)
$$

Ansatz for even solutions. Our existence proof relies upon a center manifold reduction. More precisely, we construct a smooth manifold that depends smoothly on the bifurcation parameters $\bar{\mu}$ and $c$ in a neighborhood of the origin. On the manifold, we find a smooth vector field, so that trajectories of the local flow yield solutions to the full traveling system. In Section 4, we will find heteroclinic orbits for the local flow on this manifold, which correspond to dislocation traveling waves in the full anisotropic Swift-Hohenberg equation (1.1). The dimension of the center manifold is equal to the dimension of $\mathcal{Y}_{*}$, hence it is equal to eight. However, we can reduce this dimension to four, by restricting to solutions which are even in $y$.

Indeed, as a consequence of the reflection invariance $y \mapsto-y$ of the modified Swift-Hohenberg equation (1.1), the subspace of even functions

$$
\mathcal{X}_{e}=\{U \in \mathcal{X} ; U(y)=U(-y)\},
$$

is an invariant subspace for the dynamical system (2.1) and the system (3.1). The restriction of the linear operator $\mathcal{A}(\mu, k, c, \beta)$ to $\mathcal{X}_{e}$ is a closed linear operator with dense and compactly embedded domain

$$
\mathcal{Y}_{e}=\{U \in \mathcal{Y} ; U(y)=U(-y)\},
$$

and the nonlinearity $\mathcal{F}: \mathcal{Y}_{e} \rightarrow \mathcal{Y}_{e}$ is well-defined and smooth. The spectrum of the restriction of $\mathcal{A}_{*}$ to $\mathcal{X}_{e}$ has the same properties (3.2) and (3.3) as the spectrum of the operator in the full space $\mathcal{X}$. However, the eigenvalue 0 is now geometrically double and algebraically quadruple, only. Associated eigenvectors and principal eigenvectors are given by

$$
E_{+\ell_{*}}+E_{-\ell_{*}}, \quad E_{+\left(\ell_{*}+1\right)}+E_{-\left(\ell_{*}+1\right)}, \quad F_{+\ell_{*}}+F_{-\ell_{*}}, \quad F_{+\left(\ell_{*}+1\right)}+F_{-\left(\ell_{*}+1\right)} .
$$


The restriction of the spectral projection $\mathcal{P}_{*}$ to the space $\mathcal{X}_{e}$ maps $\mathcal{X}_{e}$ onto the subspace $\mathcal{Y}_{*} \cap \mathcal{Y}_{e}$, spanned by the four vectors above.

Restricting to solutions which are even in $y$, we set

$$
\begin{aligned}
U(x, y)= & a_{0}(x)\left(E_{+\ell_{*}}(y)+E_{-\ell_{*}}(y)\right)+b_{0}(x)\left(E_{+\left(\ell_{*}+1\right)}(y)+E_{-\left(\ell_{*}+1\right)}(y)\right) \\
& +a_{1}(x)\left(F_{+\ell_{*}}(y)+F_{-\ell_{*}}(y)\right)+b_{1}(x)\left(F_{+\left(\ell_{*}+1\right)}(y)+F_{-\left(\ell_{*}+1\right)}(y)\right)+V(x, y),
\end{aligned}
$$

in which $a_{0}, a_{1}, b_{0}, b_{1}$ are real-valued functions depending upon $x$ and $V$ satisfies

$$
\mathcal{P}_{*} V(x, \cdot)=0, \quad \text { for all } x \in \mathbb{R},
$$

or, equivalently, $V(x, \cdot) \in\left(\right.$ id $\left.-\mathcal{P}_{*}\right) \mathcal{Y}_{e}$. We next substitute the ansatz (3.4) into the system (3.1), then take the scalar product with $E_{+\ell_{*}}^{\mathrm{ad}}, F_{+\ell_{*}}^{\mathrm{ad}}, E_{+\left(\ell_{*}+1\right)}^{\mathrm{ad}}, F_{+\left(\ell_{*}+1\right)}^{\mathrm{ad}}$, and project with id $-\mathcal{P}_{*}$. As a result, we find the following system of differential equations for $a_{0}, a_{1}, b_{0}, b_{1}$, and $V$ :

$$
\begin{aligned}
a_{0}^{\prime} & =a_{1} \\
a_{1}^{\prime} & =-\frac{1}{\beta-2 \sqrt{\mu_{*}}}\left(\bar{\mu} a_{0}+c a_{1}-3 a_{0}\left(a_{0}^{2}+2 b_{0}^{2}\right)\right)+\mathcal{F}_{a}^{*}\left(a_{0}, a_{1}, b_{0}, b_{1}, V, \bar{\mu}, c\right) \\
b_{0}^{\prime} & =b_{1} \\
b_{1}^{\prime} & =-\frac{1}{\beta+2 \sqrt{\mu_{*}}}\left(\bar{\mu} b_{0}+c b_{1}-3 b_{0}\left(2 a_{0}^{2}+b_{0}^{2}\right)\right)+\mathcal{F}_{b}^{*}\left(a_{0}, a_{1}, b_{0}, b_{1}, V, \bar{\mu}, c\right) \\
V_{x} & =\mathcal{A}_{*} V+\mathcal{F}_{V}^{*}\left(a_{0}, a_{1}, b_{0}, b_{1}, V, \bar{\mu}, c\right) .
\end{aligned}
$$

Here, the primes denote derivatives with respect to $x$, and

$$
\mathcal{F}_{a}^{*}, \mathcal{F}_{b}^{*}: \mathbb{R}^{4} \times\left(\mathrm{id}-\mathcal{P}_{*}\right) \mathcal{Y}_{e} \times \mathbb{R}^{2} \rightarrow \mathbb{R}, \quad \mathcal{F}_{V}^{*}: \mathbb{R}^{4} \times\left(\mathrm{id}-\mathcal{P}_{*}\right) \mathcal{Y}_{e} \times \mathbb{R}^{2} \rightarrow\left(\mathrm{id}-\mathcal{P}_{*}\right) \mathcal{Y}_{e}
$$

are smooth maps. Taking into account the estimate $\left\|\mathcal{P}_{*}\right\|_{\mathcal{L}(\mathcal{Y}, \mathcal{Y})}=\mathcal{O}(1)$ and the fact that

$$
\|u\|_{\infty}:=\sup |u(x)|=\mathcal{O}\left(k_{*}^{-1 / 2}\|u\|_{1}\right)=\mathcal{O}\left(\mu_{*}^{-1 / 4}\|u\|_{1}\right),
$$

for all $u \in H_{\mathrm{per}}^{1}(0,2 \pi)$, due to our choice of the norm of $H_{\mathrm{per}}^{1}(0,2 \pi)$, a direct calculation gives

$$
\left|\mathcal{F}_{a, b}^{*}\left(a_{0}, a_{1}, b_{0}, b_{1}, V, \bar{\mu}, c\right)\right|=\mathcal{O}\left(\left(|\bar{\mu}|+|c|+\left\|\left(a_{0}, b_{0}\right)\right\|^{2}+\mu_{*}^{-1 / 4}\left\|\left(a_{0}, b_{0}\right)\right\|\|V\|_{\mathcal{X}}+\mu_{*}^{-1 / 2}\|V\|_{\mathcal{X}}^{2}\right)\|V\|_{\mathcal{X}}\right)
$$

and

$$
\begin{aligned}
\left\|\mathcal{F}_{V}^{*}\left(a_{0}, a_{1}, b_{0}, b_{1}, V, \bar{\mu}, c\right)\right\|_{\mathcal{Y}} & =\mathcal{O}\left(|\bar{\mu}|\left\|\left(a_{0}, b_{0}\right)\right\|+|c|\left\|\left(a_{1}, b_{1}\right)\right\|+\left\|\left(a_{0}, b_{0}\right)\right\|^{3}\right. \\
& \left.+\left(|\bar{\mu}|+|c|+\left\|\left(a_{0}, b_{0}\right)\right\|^{2}+\mu_{*}^{-1 / 4}\left\|\left(a_{0}, b_{0}\right)\right\|\|V\|_{\mathcal{X}}+\mu_{*}^{-1 / 2}\|V\|_{\mathcal{X}}^{2}\right)\|V\|_{\mathcal{X}}\right) .
\end{aligned}
$$

Reduction to a center manifold. The non-uniform bounds for $\mathcal{F}_{a}, \mathcal{F}_{b}$, and $\mathcal{F}_{V}$ above, imply that we cannot expect to control the size of the center manifold uniformly when $\mu_{*} \rightarrow 0$ for the system (3.5). Furthermore, it turns out that the resolvent of $\mathcal{A}_{*}$ also satisfies a non-uniform estimate in $\mu_{*}$,

$$
\left\|\left(\mathrm{i} \omega-\mathcal{A}_{*}\right)^{-1}\right\|_{\mathcal{L}\left(\left(\mathrm{id}-\mathcal{P}_{*}\right) \mathcal{Y}\right)} \leqslant \frac{C}{\mu_{*}+|\omega|}, \quad \text { for all } \omega \in \mathbb{R},
$$


for some positive constant $C$ which does not depend upon $\mu_{*}$ (see Lemma 3.1 below). Uniform estimates for both the resolvent of the linear operator and the nonlinear terms of the system, as $\mu_{*} \rightarrow 0$, allow to control the size of the center manifold uniformly when $\mu_{*} \rightarrow 0$.

It is therefore convenient to scale variables and parameters before performing the actual reduction,

$$
\widetilde{x}=\mu_{*} x, \quad \bar{\mu}=\mu_{*}^{2} \widetilde{\bar{\mu}}, \quad c=\mu_{*} \widetilde{c}, \quad\left(a_{0}, b_{0}\right)=\mu_{*}\left(\widetilde{a}_{0}, \widetilde{b}_{0}\right), \quad\left(a_{1}, b_{1}\right)=\mu_{*}^{2}\left(\widetilde{a}_{1}, \widetilde{b}_{1}\right), \quad V=\mu_{*}^{2} \widetilde{V}, \quad j=0,1 .
$$

This scaling leads to a new system, which, after dropping the tilde (for notational simplicity), can be written in the form

$$
\begin{aligned}
a_{0}^{\prime} & =a_{1} \\
a_{1}^{\prime} & =-\frac{1}{\beta-2 \sqrt{\mu_{*}}}\left(\bar{\mu} a_{0}+c a_{1}-3 a_{0}\left(a_{0}^{2}+2 b_{0}^{2}\right)\right)+\mathcal{G}_{a}^{*}\left(a_{0}, a_{1}, b_{0}, b_{1}, V, \bar{\mu}, c\right) \\
b_{0}^{\prime} & =b_{1} \\
b_{1}^{\prime} & =-\frac{1}{\beta+2 \sqrt{\mu_{*}}}\left(\bar{\mu} b_{0}+c b_{1}-3 b_{0}\left(2 a_{0}^{2}+b_{0}^{2}\right)\right)+\mathcal{G}_{b}^{*}\left(a_{0}, a_{1}, b_{0}, b_{1}, V, \bar{\mu}, c\right) \\
V_{x} & =\frac{1}{\mu_{*}} \mathcal{A}_{*} V+\mathcal{G}_{V}^{*}\left(a_{0}, a_{1}, b_{0}, b_{1}, V, \bar{\mu}, c\right) .
\end{aligned}
$$

Here,

$\left|\mathcal{G}_{a, b}^{*}\left(a_{0}, a_{1}, b_{0}, b_{1}, V, \bar{\mu}, c\right)\right|=\mathcal{O}\left(\left(\mu_{*}|\bar{\mu}|+|c|+\mu_{*}\left\|\left(a_{0}, b_{0}\right)\right\|^{2}+\mu_{*}^{7 / 4}\left\|\left(a_{0}, b_{0}\right)\right\|\|V\|_{\mathcal{X}}+\mu_{*}^{5 / 2}\|V\|_{\mathcal{X}}^{2}\right)\|V\|_{\mathcal{X}}\right)$,

and

$$
\begin{aligned}
\left\|\mathcal{G}_{V}^{*}\left(a_{0}, a_{1}, b_{0}, b_{1}, V, \bar{\mu}, c\right)\right\|_{\mathcal{Y}} & =\mathcal{O}\left(|\bar{\mu}|\left\|\left(a_{0}, b_{0}\right)\right\|+|c|\left\|\left(a_{1}, b_{1}\right)\right\|+\left\|\left(a_{0}, b_{0}\right)\right\|^{3}\right. \\
& \left.+\left(\mu_{*}|\bar{\mu}|+|c|+\mu_{*}\left\|\left(a_{0}, b_{0}\right)\right\|^{2}+\mu_{*}^{7 / 4}\left\|\left(a_{0}, b_{0}\right)\right\|\|V\|_{\mathcal{X}}+\mu_{*}^{5 / 2}\|V\|_{\mathcal{X}}^{2}\right)\|V\|_{\mathcal{X}}\right) .
\end{aligned}
$$

Notice that the linear operator $\mathcal{A}_{*}$ is now replaced by $\frac{1}{\mu_{*}} \mathcal{A}_{*}$, for which we have the following uniform estimate on the resolvent.

Lemma 3.1 There exists a positive constant $C$, such that

$$
\left\|\left(\mathrm{i} \omega-\frac{1}{\mu_{*}} \mathcal{A}_{*}\right)^{-1}\right\|_{\mathcal{L}\left(\left(\mathrm{id}-\mathcal{P}_{*}\right) \mathcal{Y}_{e}\right)} \leqslant \frac{C}{1+|\omega|}, \quad \text { for all } \omega \in \mathbb{R}
$$

for any $\mu_{*}$ sufficiently small.

Proof. First, notice that it is enough to prove the estimate (3.6), since

$$
\left\|\left(\mathrm{i} \omega-\frac{1}{\mu_{*}} \mathcal{A}_{*}\right)^{-1}\right\|_{\mathcal{L}\left(\left(\mathrm{id}-\mathcal{P}_{*}\right) \mathcal{Y}_{e}\right)}=\mu_{*}\left\|\left(i \omega \mu_{*}-\mathcal{A}_{*}\right)^{-1}\right\|_{\mathcal{L}\left(\left(\mathrm{id}-\mathcal{P}_{*}\right) \mathcal{Y}\right)} \leqslant \frac{C \mu_{*}}{\mu_{*}+\mu_{*}|\omega|} \leqslant \frac{C}{1+|\omega|},
$$

if (3.6) holds. 
Using Fourier series and taking into account the definition of $\mathcal{P}_{*}$ we are led to estimate, uniformly in $\ell \in \mathbb{Z}, \omega \in \mathbb{R}$, and $\mu_{*}$ sufficiently small, the norm of the matrix $\left(\mathrm{i} \omega-\mathcal{A}_{*, \ell}\right)^{-1}$, where

$$
\mathcal{A}_{*, \ell}=\left(\begin{array}{cccc}
0 & 1 & 0 & 0 \\
K^{2}-1 & 0 & 1 & 0 \\
0 & 0 & 0 & 1 \\
\beta K^{2}-\beta+\mu_{*} & 0 & \beta+K^{2}-1 & 0
\end{array}\right), \quad K=k_{*}|\ell|
$$

for $\ell \in \mathbb{Z} \backslash\left\{ \pm \ell_{*}, \pm\left(\ell_{*}+1\right)\right\}$, and the norm of the restriction of $\left(\mathrm{i} \omega-\mathcal{A}_{*, \kappa}\right)^{-1}, \kappa \in\left\{ \pm \ell_{*}, \pm\left(\ell_{*}+1\right)\right\}$, to the two-dimensional spectral space associated with the non-zero eigenvalues of $\mathcal{A}_{*, \kappa}, \kappa \in\left\{ \pm \ell_{*}, \pm\left(\ell_{*}+1\right)\right\}$. Here, $\mathbb{C}^{4}$ is equipped with the norm induced by the norm of $\mathcal{Y}$, i.e., for $U_{\ell}=\left(u_{1, \ell}, u_{2, \ell}, u_{3, \ell}, u_{4, \ell}\right) \in \mathbb{C}^{4}$, we have

$$
\|U\|_{*}^{2}=\left(1+\left|k_{*}^{4} \ell^{4}\right|^{2}\right)\left|u_{1, \ell}\right|^{2}+\left(1+\left|k_{*}^{3} \ell^{3}\right|^{2}\right)\left|u_{2, \ell}\right|^{2}+\left(1+\left|k_{*}^{2} \ell^{2}\right|^{2}\right)\left|u_{3, \ell}\right|^{2}+\left(1+\left|k_{*} \ell\right|^{2}\right)\left|u_{4, \ell}\right|^{2} .
$$

Alternatively, we can equip $\mathbb{C}^{4}$ with an equivalent norm, and a convenient choice here is to take

$$
\|U\|_{*}^{2}=\left|\left(1+k_{*}|\ell|\right)^{4} u_{1, \ell}\right|^{2}+\left|\left(1+k_{*}|\ell|\right)^{3} u_{2, \ell}\right|^{2}+\left|\left(1+k_{*}|\ell|\right)^{2} u_{3, \ell}\right|^{2}+\left|\left(1+k_{*}|\ell|\right) u_{4, \ell}\right|^{2} .
$$

Then the norm of the matrix $\left(\mathrm{i} \omega-\mathcal{A}_{*, \ell}\right)^{-1}$ is the norm of the modified matrix $\left(\mathrm{i} \omega-\widetilde{\mathcal{A}}_{*, \ell}\right)^{-1}$,

$$
\widetilde{\mathcal{A}}_{*, \ell}=\left(\begin{array}{cccc}
0 & 1+K & 0 & 0 \\
\frac{1}{1+K}\left(K^{2}-1\right) & 0 & 1+K & 0 \\
0 & 0 & 0 & 1+K \\
\frac{1}{(1+K)^{3}}\left(\beta K^{2}-\beta+\mu_{*}\right) & 0 & \frac{1}{1+K}\left(\beta+K^{2}-1\right) & 0
\end{array}\right), \quad K=k_{*}|\ell|,
$$

when $\mathbb{C}^{4}$ is equipped with the standard euclidean norm.

A MAPLE computation gives

$$
\left(\mathrm{i} \omega-\widetilde{\mathcal{A}}_{*, \ell}\right)^{-1}=\frac{1}{\Delta}\left(V_{1}, V_{2}, V_{3}, V_{4}\right), \quad \Delta=\left(\omega^{2}+K^{2}-1\right)^{2}+\beta \omega^{2}-\mu_{*},
$$

where

$$
\begin{aligned}
& V_{1}=\left(\begin{array}{c}
-\mathrm{i} \omega\left(\omega^{2}+K^{2}-1+\beta\right) \\
-\frac{\left(K^{2}-1\right)\left(K^{2}-1+\omega^{2}\right)-\mu_{*}}{1+K} \\
\frac{\mathrm{i} \omega\left(\beta K^{2}-\beta+\mu_{*}\right)}{(1+K)^{2}} \\
-\frac{\omega^{2}\left(\beta K^{2}-\beta+\mu_{*}\right)}{(1+K)^{3}}
\end{array}\right), \quad V_{2}=\left(\begin{array}{c}
-(1+K)\left(\omega^{2}+K^{2}-1+\beta\right) \\
-\mathrm{i} \omega\left(K^{2}+\omega^{2}-1+\beta\right) \\
\frac{\beta K^{2}-\beta+\mu_{*}}{1+K} \\
\frac{\mathrm{i} \omega\left(\beta K^{2}-\beta+\mu_{*}\right)}{(1+K)^{2}} \\
(1+K)^{3} \\
-\omega^{2}(1+K) \\
-\mathrm{i} \omega\left(\omega^{2}+K^{2}-1\right) \\
V_{3}=\left(\begin{array}{c}
\mathrm{i} \omega(1+K)^{2} \\
-\frac{\left(K^{2}-1\right)\left(K^{2}-1+\omega^{2}\right)+\beta \omega^{2}-\mu_{*}}{1+K}
\end{array}\right), \quad V_{4}=\left(\begin{array}{c}
1+K)\left(\omega^{2}+K^{2}-1\right) \\
-\mathrm{i} \omega\left(\omega^{2}+K^{2}-1\right)
\end{array}\right),
\end{array}\right.
\end{aligned}
$$

Estimating directly the coefficients of the matrix $\left(i \omega-\widetilde{\mathcal{A}}_{*, \ell}\right)^{-1}$ gives the desired result for $\ell \in \mathbb{Z} \backslash$ $\left\{ \pm \ell_{*}, \pm\left(\ell_{*}+1\right)\right\}$.

For $\kappa \in\left\{ \pm \ell_{*}, \pm\left(\ell_{*}+1\right)\right\}$, the norm of $\mathbb{C}^{4}$ induced by the norm of $\mathcal{Y}$ is equivalent, uniformly as $\mu_{*} \rightarrow 0$, with the euclidean norm of $\mathbb{C}^{4}$, since $k_{*} \ell_{*}=\mathcal{O}(1)$ and $k_{*}\left(\ell_{*}+1\right)=\mathcal{O}(1)$, as $\mu_{*} \rightarrow 0$. Then the desired estimate is a consequence of the fact that the two nonzero eigenvalues of $\mathcal{A}_{*, \kappa}$, for $\kappa \in\left\{ \pm \ell_{*}, \pm\left(\ell_{*}+1\right)\right\}$, are both simple and $\mathcal{O}(1)$, as $\mu_{*} \rightarrow 0$. This completes the proof of the lemma. 
We are now ready to apply the center manifold theorem as stated for instance in [6]. We find three neighborhoods of the origin, $\mathcal{U} \subset \mathbb{R}^{4}, \mathcal{V}_{*} \subset\left(\right.$ id $\left.-\mathcal{P}_{*}\right) \mathcal{Y}_{e}, \mathcal{W} \subset \mathbb{R}^{2}$, and a map $h_{*}: \mathcal{U} \times \mathcal{W} \rightarrow \mathcal{V}_{*}$ of class $C^{k}$, for an arbitrary, but fixed $k \geqslant 1$, with estimates

$$
\left\|h_{*}\left(a_{0}, a_{1}, b_{0}, b_{1}, \bar{\mu}, c\right)\right\| \mathcal{Y}=\mathcal{O}\left(|\bar{\mu}|\left\|\left(a_{0}, b_{0}\right)\right\|+|c|\left\|\left(a_{1}, b_{1}\right)\right\|+\left\|\left(a_{0}, b_{0}\right)\right\|^{3}\right)
$$

such that for any $(\bar{\mu}, c) \in \mathcal{W}$, all bounded solutions of $(3.7)$ with $\left(a_{0}, a_{1}, b_{0}, b_{1}, V\right)(x) \in \mathcal{U} \times \mathcal{V}_{*}$, for all $x \in \mathbb{R}$, satisfy

$$
V(x)=h_{*}\left(a_{0}(x), a_{1}(x), b_{0}(x), b_{1}(x), \bar{\mu}, c\right), \quad \text { for all } x \in \mathbb{R} .
$$

Due to the uniform estimates on the resolvent in Lemma 3.1 and of the nonlinear terms $\mathcal{G}_{a, b}^{*}$ and $\mathcal{G}_{V}^{*}$, the neighborhoods $\mathcal{U}$ and $\mathcal{W}$ can be chosen independent of $\mu_{*}$, and $\mathcal{V}_{*}$ is of size $\mathcal{O}(1)$, as $\mu_{*} \rightarrow 0$. Moreover, the estimate on $h_{*}$ is uniform, as $\mu_{*} \rightarrow 0$.

Substituting (3.8) into (3.7), we obtain the reduced system

$$
\begin{aligned}
a_{0}^{\prime} & =a_{1} \\
a_{1}^{\prime} & =-\frac{1}{\beta-2 \sqrt{\mu_{*}}}\left(\bar{\mu} a_{0}+c a_{1}-3 a_{0}\left(a_{0}^{2}+2 b_{0}^{2}\right)\right)+\mathcal{R}_{a}^{*}\left(a_{0}, a_{1}, b_{0}, b_{1}, \bar{\mu}, c\right) \\
b_{0}^{\prime} & =b_{1} \\
b_{1}^{\prime} & =-\frac{1}{\beta+2 \sqrt{\mu_{*}}}\left(\bar{\mu} b_{0}+c b_{1}-3 b_{0}\left(2 a_{0}^{2}+b_{0}^{2}\right)\right)+\mathcal{R}_{b}^{*}\left(a_{0}, a_{1}, b_{0}, b_{1}, \bar{\mu}, c\right)
\end{aligned}
$$

where

$$
\left|\mathcal{R}_{a, b}^{*}\left(a_{0}, a_{1}, b_{0}, b_{1}, \bar{\mu}, c\right)\right|=\mathcal{O}\left(\left(\mu_{*}|\bar{\mu}|+|c|+\mu_{*}\left\|\left(a_{0}, b_{0}\right)\right\|^{2}\right)\left(|\bar{\mu}|\left\|\left(a_{0}, b_{0}\right)\right\|+|c|\left\|\left(a_{1}, b_{1}\right)\right\|+\left\|\left(a_{0}, b_{0}\right)\right\|^{3}\right)\right) .
$$

Symmetries. The modified Swift-Hohenberg (1.2) possesses three reflection symmetries

$$
u \mapsto-u, \quad y \mapsto-y, \quad(x, c) \mapsto(-x,-c)
$$

These symmetries are inherited by the dynamical system (2.1), and are preserved in the ansatz (3.4) and in the center manifold reduction in the following sense. The symmetry induced by the reflection $y \mapsto-y$ acts trivially on the reduced system (3.9), because of the restriction to functions which are even in $y$. The reflection $u \mapsto-u$ implies that the vector field in the reduced system (3.9) commutes with

$$
\mathcal{S}\left(a_{0}, a_{1}, b_{0}, b_{1}\right)=\left(-a_{0},-a_{1},-b_{0},-b_{1}\right) .
$$

The reflection $(x, c) \mapsto(-x,-c)$ implies that the vector field in the reduced system $(3.9)$ is reversible, i.e., it anticommutes with

$$
\mathcal{R}\left(a_{0}, a_{1}, b_{0}, b_{1} ; c\right)=\left(a_{0},-a_{1}, b_{0},-b_{1} ;-c\right) .
$$

Remark 3.2 Without the restriction to even solutions, the ansatz (3.4) becomes

$$
\begin{aligned}
U(x, y)= & a_{0}(x) E_{+\ell_{*}}(y)+\overline{a_{0}}(x) E_{-\ell_{*}}(y)+a_{1}(x) E_{+\left(\ell_{*}+1\right)}(y)+\overline{a_{1}}(x) E_{-\left(\ell_{*}+1\right)}(y) \\
& +b_{0}(x) F_{+\ell_{*}}(y)+\overline{b_{0}}(x) F_{-\ell_{*}}(y)+b_{1}(x) F_{+\left(\ell_{*}+1\right)}(y)+\overline{b_{1}}(x) F_{-\left(\ell_{*}+1\right)}(y)+V(x, y),
\end{aligned}
$$


in which $a_{0}, a_{1}, b_{0}, b_{1}$ are complex-valued functions depending upon $x$, and $V$ satisfies $\mathcal{P}_{*} V=0$. Now, the center manifold theorem leads to the reduced system

$$
\begin{aligned}
a_{0}^{\prime} & =a_{1} \\
a_{1}^{\prime} & =-\frac{1}{\beta-2 \sqrt{\mu_{*}}}\left(\bar{\mu} a_{0}+c a_{1}-3 a_{0}\left(\left|a_{0}\right|^{2}+2\left|b_{0}\right|^{2}\right)\right)+\mathcal{R}_{a}^{*}\left(a_{0}, a_{1}, b_{0}, b_{1}, \bar{\mu}, c\right) \\
b_{0}^{\prime} & =b_{1} \\
b_{1}^{\prime} & =-\frac{1}{\beta+2 \sqrt{\mu_{*}}}\left(\bar{\mu} b_{0}+c b_{1}-3 b_{0}\left(2\left|a_{0}\right|^{2}+\left|b_{0}\right|^{2}\right)\right)+\mathcal{R}_{b}^{*}\left(a_{0}, a_{1}, b_{0}, b_{1}, \bar{\mu}, c\right) .
\end{aligned}
$$

As a consequence of the invariance of the modified Swift-Hohenberg equation (1.2) under translations in $y$, this reduced system is equivariant under the actions of the circle group

$$
\mathcal{R}_{\varphi}\left(a_{0}, a_{1}, b_{0}, b_{1}\right)=\left(\mathrm{e}^{\mathrm{i} \ell_{\star} \varphi} a_{0}, \mathrm{e}^{\mathrm{i} \ell_{\star} \varphi} a_{1}, \mathrm{e}^{\mathrm{i}\left(\ell_{\star}+1\right) \varphi} b_{0}, \mathrm{e}^{\mathrm{i}\left(\ell_{\star}+1\right) \varphi} b_{1}\right), \quad \varphi \in \mathbb{R} / 2 \pi \mathbb{Z} .
$$

The different rotation number of the circle group in a- and b-components effectively forces the Taylor jet to be invariant under the torus action $\operatorname{diag}\left(\mathrm{e}^{\mathrm{i} \psi_{1}}, \mathrm{e}^{\mathrm{i} \psi_{1}}, \mathrm{e}^{\mathrm{i} \psi_{2}}, \mathrm{e}^{\mathrm{i} \psi_{2}}\right),\left(\psi_{1}, \psi_{2}\right) \in[0,2 \pi)^{2}$ up to order $2 \ell_{\star}-1$,

when terms of the form $a_{j}^{\ell_{\star}} \bar{b}_{j}^{\ell_{\star}-1}$ appear and break this symmetry. As a consequence, our analysis would predict dislocations with arbitrary shifts of the rolls with wavenumber $\ell_{\star}+1$ relative to the rolls with wavenumber $\ell_{\star}$, up to order $2 \ell_{\star}-2$. However only even solutions can be shown to persist beyond that order.

The special translation by half the domain width $\varphi=\pi$ leaves the subspace of even functions invariant and therefore induces a symmetry of the reduced system. This symmetry corresponds to a reflection $\left(a_{0}, a_{1}, b_{0}, b_{1}\right) \mapsto\left(-a_{0},-a_{1}, b_{0}, b_{1}\right)$ for $\ell_{\star}$ odd and $\left(a_{0}, a_{1}, b_{0}, b_{1}\right) \mapsto\left(a_{0}, a_{1},-b_{0},-b_{1}\right)$ for $\ell_{\star}$ even. Together with the symmetry $u \mapsto-u$, both cases, $\ell_{\star}$ even or odd, yield the same symmetry group, generated by both the aforementioned reflections.

\section{Heteroclinics in the reduced system}

In this section we show that the reduced system (3.9) possesses heteroclinic orbits, which correspond to dislocations for the modified Swift-Hohenberg equation (1.1).

The reduced system (3.9) is equivalent with the following system of second order ordinary differential equations

$$
\begin{aligned}
a_{0}^{\prime \prime} & =-\frac{1}{\beta-2 \sqrt{\mu_{*}}}\left(\bar{\mu} a_{0}+c a_{0}^{\prime}-3 a_{0}\left(a_{0}^{2}+2 b_{0}^{2}\right)\right)+\mathcal{R}_{a}^{*}\left(a_{0}, a_{0}^{\prime}, b_{0}, b_{0}^{\prime}, \bar{\mu}, c\right) \\
b_{0}^{\prime \prime} & =-\frac{1}{\beta+2 \sqrt{\mu_{*}}}\left(\bar{\mu} b_{0}+c b_{0}^{\prime}-3 b_{0}\left(2 a_{0}^{2}+b_{0}^{2}\right)\right)+\mathcal{R}_{b}^{*}\left(a_{0}, a_{0}^{\prime}, b_{0}, b_{0}^{\prime}, \bar{\mu}, c\right) .
\end{aligned}
$$

Assuming that $\bar{\mu}>0$, we introduce the scaled variables

$$
X=\frac{1}{\sqrt{\beta}} \sqrt{\bar{\mu}} x, \quad c=\sqrt{\bar{\mu}} \bar{c}, \quad a_{0}=\frac{1}{\sqrt{3}} \sqrt{\bar{\mu}} A_{0}, \quad b_{0}=\frac{1}{\sqrt{3}} \sqrt{\bar{\mu}} B_{0},
$$

which leads to the system

$$
\begin{aligned}
& A_{0}^{\prime \prime}=-A_{0}-\bar{c} A_{0}^{\prime}+A_{0}\left(A_{0}^{2}+2 B_{0}^{2}\right)+\mathcal{R}_{A}^{*}\left(A_{0}, A_{0}^{\prime}, B_{0}, B_{0}^{\prime}, \bar{\mu}, \bar{c} ; \mu_{*}\right) \\
& B_{0}^{\prime \prime}=-B_{0}-\bar{c} B_{0}^{\prime}+B_{0}\left(2 A_{0}^{2}+B_{0}^{2}\right)+\mathcal{R}_{B}^{*}\left(A_{0}, A_{0}^{\prime}, B_{0}, B_{0}^{\prime}, \bar{\mu}, \bar{c} ; \mu_{*}\right),
\end{aligned}
$$


with

$$
\mathcal{R}_{A, B}^{*}\left(A_{0}, A_{0}^{\prime}, B_{0}, B_{0}^{\prime}, \bar{\mu}, \bar{c}\right)=\mathcal{O}\left(\mu_{*}^{1 / 2}+\bar{\mu}^{1 / 2}\right)
$$

\subsection{The leading order system}

First, we consider the truncated system

$$
\begin{aligned}
& A_{0}^{\prime \prime}=-A_{0}+A_{0}\left(A_{0}^{2}+2 B_{0}^{2}\right), \\
& B_{0}^{\prime \prime}=-B_{0}+B_{0}\left(2 A_{0}^{2}+B_{0}^{2}\right),
\end{aligned}
$$

obtained by setting $\mu_{*}=\bar{\mu}=\bar{c}=0$ in (4.2). The existence of heteroclinic connections for this system has been studied in a different context in [12, Section 1.2]. The system possesses 9 equilibria

$$
(0,0), \quad(0, \pm 1), \quad( \pm 1,0), \quad\left( \pm \frac{1}{\sqrt{3}}, \pm \frac{1}{\sqrt{3}}\right) .
$$

There are three different types of heteroclinic orbits that connect these equilibria:

- two heteroclinic orbits of the form

$$
\left(A_{0}(x), 0\right), \quad\left(0, B_{0}(x)\right)
$$

connecting the single-mode equilibria $( \pm 1,0)$ and $(0, \pm 1)$, respectively;

- two symmetric heteroclinic orbits of the form

$$
\left(A_{0}(x), A_{0}(x)\right), \quad\left(A_{0}(x),-A_{0}(x)\right)
$$

connecting the bimodal equilibria $\left( \pm \frac{1}{\sqrt{3}}, \pm \frac{1}{\sqrt{3}}\right)$ and $\left( \pm \frac{1}{\sqrt{3}}, \mp \frac{1}{\sqrt{3}}\right)$, respectively;

- four heteroclinic orbits of the form

$$
\left(A_{0}(x), B_{0}(x)\right), \quad\left(A_{0}(x),-B_{0}(x)\right), \quad\left(-A_{0}(x), B_{0}(x)\right), \quad\left(-A_{0}(x),-B_{0}(x)\right),
$$

connecting the single-mode equilibria $(1,0)$ with $(0,1),(1,0)$ with $(0,-1),(-1,0)$ with $(0,1)$, and $(-1,0)$ with $(0,-1)$, respectively.

Of interest for us are the heteroclinic orbits of the third type, since these correspond to dislocations for the modified Swift-Hohenberg equation (1.1). While the existence of the first two types of heteroclinic orbits is straightforward, as they all lie in some two-dimensional invariant subspace, the proof of existence of a heteroclinic orbit of the third type is more complicated. Relying upon a variational method, the following result has been proved in [12, Theorem 5].

Lemma 4.1 (Existence of a heteroclinic orbit [12]) The system (4.3) possesses a smooth solution $\left(A_{0}^{\star}, B_{0}^{\star}\right) \in C^{\infty}\left(\mathbb{R}, \mathbb{R}^{2}\right)$ with the following properties:

(i) $\lim _{x \rightarrow-\infty}\left(A_{0}^{\star}(x), B_{0}^{\star}(x)\right)=(1,0)$ and $\lim _{x \rightarrow \infty}\left(A_{0}^{\star}(x), B_{0}^{\star}(x)\right)=(0,1)$; 
(ii) $A_{0}^{\star}(x) \geqslant 0$ and $B_{0}^{\star}(x) \geqslant 0$, for all $x \in \mathbb{R}$;

(iii) $A_{0}^{\star}(x)=B_{0}^{\star}(-x)$, for all $x \in \mathbb{R}$;

(iv) $A_{0}^{\star}(x)=B_{0}^{\star}(x)$ if and only if $x=0$;

(v) $A_{0}^{\star 2}(x)+B_{0}^{\star 2}(x) \leqslant 1$ and $A_{0}^{\star}(x)+B_{0}^{\star}(x) \geqslant 1$, for all $x \in \mathbb{R}$;

(vi) the angular coordinate $\varphi_{\star}=\arctan \frac{B_{0}^{\star}}{A_{0}^{\star}}$ increases monotonically, i.e., $\varphi_{\star}^{\prime}(x) \geqslant 0$, for all $x \in \mathbb{R}$.

We will need some additional properties that we state in the following lemma.

Lemma 4.2 (Properties of the heteroclinic orbit) The functions $A_{0}^{\star}$ and $B_{0}^{\star}$ in Lemma 4.1 are strictly monotone. More precisely, we have

$$
A_{0}^{\star \prime}(x)<0, \quad B_{0}^{\star \prime}(x)>0, \quad \text { for all } x \in \mathbb{R},
$$

and

$$
0<A_{0}^{\star}(x)<1, \quad 0<B_{0}^{\star}(x)<1, \quad \text { for all } x \in \mathbb{R} \text {. }
$$

Proof. First, we show the inequalities (4.5). The inequalities (ii) and (v) in Lemma 4.1 imply that

$$
0 \leqslant A_{0}^{\star}(x) \leqslant 1, \quad 0 \leqslant B_{0}^{\star}(x) \leqslant 1, \quad \text { for all } x \in \mathbb{R} .
$$

Assume that $A_{0}^{\star}\left(x_{0}\right)=0$, for some $x_{0} \in \mathbb{R}$. Then, the inequalities (v) in Lemma 4.1 imply that $B_{0}^{\star}\left(x_{0}\right)=1$, so that $x_{0}$ is a critical point of both $A_{0}^{\star}$ and $B_{0}^{\star}$, i.e.,

$$
A_{0}^{\star \prime}\left(x_{0}\right)=B_{0}^{\star \prime}\left(x_{0}\right)=0 .
$$

By uniqueness of solutions of the system of differential equations (4.3), it follows that

$$
A_{0}^{\star}(x)=0, \quad B_{0}^{\star}(x)=1, \quad \text { for all } x \in \mathbb{R},
$$

which contradicts property (i) in Lemma 4.1. Consequently, $A_{0}^{\star}(x)>0$, for all $x \in \mathbb{R}$. The other inequalities in (4.5) are obtained in a similar fashion.

We show (4.4), next. Notice that it is enough to show the first inequality in (4.4). The symmetry property (iii) in Lemma 4.1 will then give the second inequality. Property (vi) in Lemma 4.1 implies that

$$
B_{0}^{\star \prime}(x) A_{0}^{\star}(x)-A_{0}^{\star \prime}(x) B_{0}^{\star}(x) \geqslant 0, \quad \text { for all } x \in \mathbb{R} .
$$

The variational structure of (4.3) yields the first integral

$$
H\left(A_{0}, B_{0}, A_{0}^{\prime}, B_{0}^{\prime}\right)=\left(A_{0}^{\prime}\right)^{2}+\left(B_{0}^{\prime}\right)^{2}-\frac{1}{2}\left(A_{0}^{2}+B_{0}^{2}-1\right)^{2}-A_{0}^{2} B_{0}^{2} .
$$

Taking into account the asymptotic behavior of $A_{0}^{\star}$ and $B_{0}^{\star}$, the conservation of $H$ along trajectories implies that

$$
\left(A_{0}^{\star \prime}\right)^{2}+\left(B_{0}^{\star \prime}\right)^{2}=\frac{1}{2}\left(A_{0}^{\star 2}+B_{0}^{\star 2}-1\right)^{2}+A_{0}^{\star 2} B_{0}^{\star 2} .
$$


Since the map

$$
\left(A_{0}, B_{0}\right) \mapsto \frac{1}{2}\left(A_{0}^{2}+B_{0}^{2}-1\right)^{2}+A_{0}^{2} B_{0}^{2}
$$

is strictly positive in the set

$$
\left\{\left(A_{0}, B_{0}\right) \in \mathbb{R}^{2} ; A_{0}>0, B_{0}>0, A_{0}+B_{0} \geqslant 1, A_{0}^{2}+B_{0}^{2} \leqslant 1\right\},
$$

we conclude that

$$
\left(A_{0}^{\star \prime}(x)\right)^{2}+\left(B_{0}^{\star \prime}(x)\right)^{2}>0, \quad \text { for all } x \in \mathbb{R} .
$$

Now assume that the first inequality in (4.4) does not hold. Then there exists $x_{0} \in \mathbb{R}$ such that

$$
A_{0}^{\star \prime}\left(x_{0}\right) \geqslant 0, \quad A_{0}^{\star \prime \prime}\left(x_{0}\right)=0, \quad A_{0}^{\star \prime \prime \prime}\left(x_{0}\right) \leqslant 0,
$$

that is, $x_{0}$ is a maximum of $A_{0}^{\star \prime}$. Since $\left(A_{0}^{\star \prime}, B_{0}^{\star \prime}\right)$ solves the linearized system

$$
\begin{aligned}
& a_{0}^{\prime \prime}=\left(-1+3 A_{0}^{\star 2}+2 B_{0}^{\star 2}\right) a_{0}+4 A_{0}^{\star} B_{0}^{\star} b_{0}, \\
& b_{0}^{\prime \prime}=\left(-1+2 A_{0}^{\star 2}+3 B_{0}^{\star 2}\right) b_{0}+4 A_{0}^{\star} B_{0}^{\star} a_{0},
\end{aligned}
$$

we conclude that

$$
\left(-1+3 A_{0}^{\star 2}\left(x_{0}\right)+2 B_{0}^{\star 2}\left(x_{0}\right)\right) A_{0}^{\star \prime}\left(x_{0}\right)+4 A_{0}^{\star}\left(x_{0}\right) B_{0}^{\star}\left(x_{0}\right) B_{0}^{\star \prime}\left(x_{0}\right) \leqslant 0 .
$$

Since

$$
-1+3 A_{0}^{\star 2}\left(x_{0}\right)+2 B_{0}^{\star 2}\left(x_{0}\right) \geqslant-1+3 A_{0}^{\star 2}\left(x_{0}\right)+2\left(1-A_{0}^{\star}\left(x_{0}\right)\right)^{2}=1-4 A_{0}^{\star}\left(x_{0}\right)+5 A_{0}^{\star 2}\left(x_{0}\right) \geqslant \frac{1}{5},
$$

we can conclude that $B_{0}^{\star \prime}\left(x_{0}\right) \leqslant 0$. This together with (4.6) implies that

$$
A_{0}^{\star \prime}\left(x_{0}\right)=B_{0}^{\star \prime}\left(x_{0}\right)=0 .
$$

This contradicts (4.8), and proves the first inequality in (4.4).

Remark 4.3 (Asymptotic behavior) The solution $\left(A_{0}^{\star}, B_{0}^{\star}\right)$ in Lemma 4.1 corresponds to a heteroclinic orbit of the dynamical system

$$
\begin{aligned}
& A_{0}^{\prime}=A_{1} \\
& A_{1}^{\prime}=-A_{0}+A_{0}\left(A_{0}^{2}+2 B_{0}^{2}\right) \\
& B_{0}^{\prime}=B_{1} \\
& B_{1}^{\prime}=-B_{0}+B_{0}\left(2 A_{0}^{2}+B_{0}^{2}\right)
\end{aligned}
$$

connecting the equilibria $(1,0,0,0)$ and $(0,0,1,0)$. Both equilibria are saddles, with a two-dimensional stable manifold and a two-dimensional unstable manifold. Consequently, the heteroclinic orbit $\left(A_{0}^{\star}, B_{0}^{\star}\right)$ belongs to the intersection of the unstable manifold of the equilibrium $(1,0,0,0)$ with the stable manifold of the equilibrium $(0,0,1,0)$. Taking into account the fact that the strong stable manifold of the $(0,0,1,0)$ belongs to the invariant subspace $\left\{\left(A_{0}, A_{1}, B_{0}, B_{1}\right) \in \mathbb{R}^{4} ; A_{0}=A_{1}=0\right\}$, this allows to derive the following asymptotic behavior for $A_{0}^{\star}$ and $B_{0}^{\star}$ as $x \rightarrow \infty$ :

$$
A_{0}^{\star}(x)=\alpha_{\star} e^{-x}+\mathcal{O}\left(e^{-(1+\delta) x}\right), \quad B_{0}^{\star}(x)=1-\beta_{\star} e^{-\sqrt{2} x}+\mathcal{O}\left(e^{-(\sqrt{2}+\delta) x}\right),
$$


with some $\delta>0$, and

$$
\alpha_{\star}>0, \quad \beta_{\star} \geqslant 0 .
$$

We will show in Remark 4.5, that, in fact, $\beta_{\star}>0$, as well. As $x \rightarrow-\infty$, the asymptotic behavior follows then from the symmetry property (iii) in Lemma 4.1,

$$
A_{0}^{\star}(x)=1-\beta_{\star} e^{\sqrt{2} x}+\mathcal{O}\left(e^{(\sqrt{2}+\delta) x}\right), \quad B_{0}^{\star}(x)=\alpha_{\star} e^{x}+\mathcal{O}\left(e^{(1+\delta) x}\right) .
$$

\subsection{Linearized system}

We consider the linearization of the truncated system $(4.3)$ at $\left(A_{0}^{\star}, B_{0}^{\star}\right)$, next. The linearization can be viewed as a closed linear operator,

$$
\mathcal{L}_{\star}=\left(\begin{array}{cc}
\partial_{x x}+1-3 A_{0}^{\star 2}-2 B_{0}^{\star 2} & -4 A_{0}^{\star} B_{0}^{\star} \\
-4 A_{0}^{\star} B_{0}^{\star} & \partial_{x x}+1-2 A_{0}^{\star 2}-3 B_{0}^{\star 2}
\end{array}\right),
$$

with dense domain in $C_{b}\left(\mathbb{R} ; \mathbb{R}^{2}\right)$. Our purpose is to show that $\mathcal{L}_{\star}$ is a Fredholm operator with index 0 , and that 0 is an algebraically simple eigenvalue of $\mathcal{L}_{\star}$.

Notice that the derivative $\left(A_{0}^{\star \prime}, B_{0}^{\star \prime}\right)$ of the heteroclinic orbit belongs to the kernel of $\mathcal{L}_{\star}$, due to the translation invariance of the truncated system (4.3). The following result describes the behavior of the elements in the kernel of $\mathcal{L}_{\star}$ for large $x$.

Lemma 4.4 Assume that $\left(a_{0}, b_{0}\right) \in C_{b}^{2}\left(\mathbb{R} ; \mathbb{R}^{2}\right)$ belongs to the kernel of $\mathcal{L}_{\star}$. If $b_{0}$ is positive (resp. negative), for sufficiently large $x$, then $a_{0}$ is negative (resp. positive), for sufficiently large $x$, and the functions $a_{0}$ and $b_{0}$ have the asymptotic behavior

$$
a_{0}(x)=\alpha_{+} e^{-x}+\mathcal{O}\left(e^{-(1+\delta) x}\right), \quad b_{0}(x)=\beta_{+} e^{-\sqrt{2} x}+\mathcal{O}\left(e^{-(\sqrt{2}+\delta) x}\right),
$$

$\alpha_{+}<0, \beta_{+}>0$ (resp. $\left.\alpha_{+}>0, \beta_{+}<0\right)$, as $x \rightarrow \infty$, for some $\delta>0$.

Proof. The kernel of $\mathcal{L}_{\star}$ consists of bounded functions $\left(a_{0}, b_{0}\right)$ which solve the first order system of linear ODEs

$$
\begin{aligned}
& a_{0}^{\prime}=a_{1} \\
& a_{1}^{\prime}=\left(-1+3 A_{0}^{\star 2}+2 B_{0}^{\star 2}\right) a_{0}+4 A_{0}^{\star} B_{0}^{\star} b_{0} \\
& b_{0}^{\prime}=b_{1} \\
& b_{1}^{\prime}=\left(-1+2 A_{0}^{\star 2}+3 B_{0}^{\star 2}\right) b_{0}+4 A_{0}^{\star} B_{0}^{\star} a_{0} .
\end{aligned}
$$

As $x \rightarrow \pm \infty$, a first approximation to solutions of this system is given by solutions of the autonomous systems

$$
U^{\prime}=\mathcal{A}_{ \pm} U, \quad \mathcal{A}_{+}=\left(\begin{array}{cccc}
0 & 1 & 0 & 0 \\
1 & 0 & 0 & 0 \\
0 & 0 & 0 & 1 \\
0 & 0 & 2 & 0
\end{array}\right), \quad \mathcal{A}_{-}=\left(\begin{array}{llll}
0 & 1 & 0 & 0 \\
2 & 0 & 0 & 0 \\
0 & 0 & 0 & 1 \\
0 & 0 & 1 & 0
\end{array}\right)
$$


The matrices $\mathcal{A}_{ \pm}$have the eigenvalues $\pm 1, \pm \sqrt{2}$, which predicts the asymptotic behavior for bounded solutions of the form

$$
a_{0}(x)=\alpha_{+} e^{-x}+\mathcal{O}\left(e^{-(1+\delta) x}\right), \quad b_{0}(x)=\beta_{+} e^{-\sqrt{2} x}+\mathcal{O}\left(e^{-(\sqrt{2}+\delta) x}\right), \quad \alpha_{+} \in \mathbb{R}, \quad \beta_{+} \in \mathbb{R},
$$

as $x \rightarrow \infty$, and

$$
a_{0}(x)=\alpha_{-} e^{\sqrt{2} x}+\mathcal{O}\left(e^{(\sqrt{2}+\delta) x}\right), \quad b_{0}(x)=\beta_{-} e^{x}+\mathcal{O}\left(e^{(1+\delta) x}\right), \quad \alpha_{-} \in \mathbb{R}, \quad \beta_{-} \in \mathbb{R},
$$

as $x \rightarrow-\infty$, for some $\delta>0$. Using exponential dichotomies and expansions, one can show that the space of solutions bounded at $x=+\infty$ is two-dimensional, and the general solution is a linear combination of a unique solution with decay $\mathrm{O}\left(\mathrm{e}^{-\sqrt{2} x}\right), \alpha_{+}=0, \beta_{+}=1$, and a solution with asymptotics $\mathrm{e}^{-x}, \alpha_{+}=1$, $\beta_{+}=0$. In particular, this shows that the functions $a_{0}$ and $b_{0}$ are exponentially decaying to 0 , as $x \rightarrow \pm \infty$, and $\alpha_{+}=\beta_{+}=0$ if and only if $\left(a_{0}, b_{0}\right)=(0,0)$.

For both solutions, one can derive exponential expansions in sums of $\mathrm{e}^{-(j+\sqrt{2} m) x}, j, m \geqslant 1$, for $x \rightarrow \infty$. Consider for instance the solution with $\beta_{+}=0$. The $b$-component solves at leading order $b^{\prime \prime}-2 b=$ $4 A_{0}^{\star} B_{0}^{\star} a$. Since $A_{0}^{\star} B_{0}^{\star}=\alpha_{\star} \mathrm{e}^{-x}\left(1+\mathrm{O}\left(\mathrm{e}^{-\delta x}\right)\right)$ with $\alpha_{\star}>0$, this gives $b=\beta \mathrm{e}^{-2 x}\left(1+\mathrm{O}\left(\mathrm{e}^{-\delta x}\right)\right)$ with $\beta=2 \alpha_{\star} \alpha_{+}$. Similarly one finds expansions for $a_{0}$ when $\alpha_{+}=0$. As a consequence, we conclude that all solutions to the linear equation are monotone for sufficiently large $x$. In particular, $a_{0}$ and $b_{0}$ both do not change sign for $x$ large enough. The same argument applies to $x \rightarrow-\infty$.

Next, assume that the second component $b_{0}$ of a function in the kernel is positive for sufficiently large $x$. (If $b_{0}$ is negative, it is enough to replace $\left(a_{0}, b_{0}\right)$ by $\left(-a_{0},-b_{0}\right)$ in the next arguments.) Since $b_{0}$ is exponentially decaying to 0 as $x \rightarrow \pm \infty$, the function $b_{0}$ has at least one local maximum. Consider the local maximum of $b_{0}$ at $x_{M}$, with $x_{M}$ maximal. We have

$$
b_{0}\left(x_{M}\right)>0, \quad b_{0}^{\prime}\left(x_{M}\right)=0, \quad b_{0}^{\prime \prime}\left(x_{M}\right) \leqslant 0,
$$

and

$$
b_{0}(x)>0, \quad \text { for all } x \geqslant x_{M} .
$$

We also have

$$
4 A_{0}^{\star}\left(x_{M}\right) B_{0}^{\star}\left(x_{M}\right) a_{0}\left(x_{M}\right)=b_{0}^{\prime \prime}\left(x_{M}\right)-\left(-1+2 A_{0}^{\star 2}\left(x_{M}\right)+3 B_{0}^{\star 2}\left(x_{M}\right)\right) b_{0}\left(x_{M}\right)<0,
$$

since

$$
-1+2 A_{0}^{\star 2}\left(x_{M}\right)+3 B_{0}^{\star 2}\left(x_{M}\right) \geqslant-1+2\left(1-B_{0}^{\star}\left(x_{M}\right)\right)^{2}+3 B_{0}^{\star 2}\left(x_{M}\right)=1-4 B_{0}^{\star}\left(x_{M}\right)+5 B_{0}^{\star 2}\left(x_{M}\right) \geqslant \frac{1}{5} .
$$

Consequently,

$$
a_{0}\left(x_{M}\right)<0 .
$$

We claim that $a_{0}(x)<0$, for any $x \geqslant x_{M}$, which would then prove that $a_{0}$ is negative, for sufficiently large $x$. Indeed, otherwise there would exist a local maximum $y_{M}>x_{M}$ of $a_{0}$ such that

$$
a_{0}\left(y_{M}\right) \geqslant 0, \quad a_{0}^{\prime}\left(y_{M}\right)=0, \quad a_{0}^{\prime \prime}\left(y_{M}\right) \leqslant 0,
$$

since $a_{0}$ is exponentially decaying as $x \rightarrow \pm \infty$. The same reasoning as above then gives that $b_{0}\left(y_{M}\right) \leqslant 0$, which contradicts (4.13). 
From these arguments, we conclude that

$$
\alpha_{+} \leqslant 0, \quad \beta_{+} \geqslant 0 .
$$

in the expansion (4.11) for $a_{0}$ and $b_{0}$. We claim that both coefficients do not vanish, which would then prove the lemma. Indeed, suppose that $\alpha_{+}=0$. Then $\beta_{+}>0$, since $\alpha_{+}$and $\beta_{+}$do not vanish simultaneously. Computing the leading-order term in the expansion for $a_{0}$ as above, we find that

$$
a_{0}(x)=\frac{2 \alpha_{\star} \beta_{+}}{1+\sqrt{2}} \mathrm{e}^{-(1+\sqrt{2}) x}\left(1+\mathrm{O}\left(\mathrm{e}^{-\delta x}\right)\right) .
$$

Since $\alpha_{\star}>0$, this implies $a_{0}>0$ for large $x$, which contradicts our starting assumption and therefore proves that $\alpha_{+} \neq 0$. The same argument can be used to exclude the case $\beta_{+}=0$. This concludes the proof of the lemma.

Remark 4.5 Since $\left(A_{0}^{\star \prime}, B_{0}^{\star \prime}\right)$ belongs to the kernel of $\mathcal{L}_{\star}$, and $B_{0}^{\star \prime}>0$, this lemma implies that $\beta_{\star}$ in (4.9) is strictly positive, $\beta_{\star}>0$.

Lemma 4.6 (Linearized operator) The linear operator $\mathcal{L}_{\star}$ acting in $C_{b}\left(\mathbb{R} ; \mathbb{R}^{2}\right)$ is Fredholm with index 0 . Furthermore, 0 is an algebraically simple eigenvalue of $\mathcal{L}_{\star}$, with associated eigenvector $\left(A_{0}^{\star \prime}, B_{0}^{\star \prime}\right)$.

Proof. The linear operator $\mathcal{L}_{\star}$ is a relatively compact perturbation of the asymptotic operator

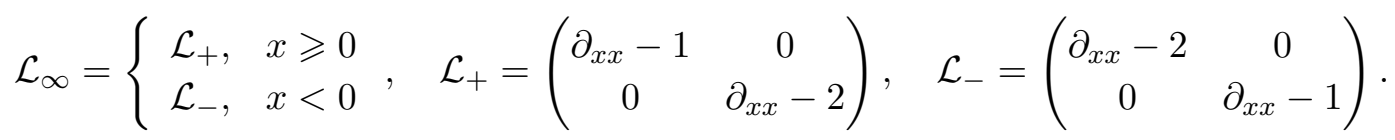

A direct calculation shows that the spectra of $\mathcal{L}_{+}$and $\mathcal{L}_{-}$are given by

$$
\sigma\left(\mathcal{L}_{+}\right)=\sigma\left(\mathcal{L}_{-}\right)=(-\infty,-1],
$$

so that the essential spectrum of $\mathcal{L}_{\infty}$, i.e., the set of $\lambda \in \mathbb{C}$ for which the operator $\lambda-\mathcal{L}_{\infty}$ is not Fredholm with index 0 , is

$$
\sigma_{\text {ess }}\left(\mathcal{L}_{\infty}\right)=(-\infty,-1]
$$

Consequently,

$$
\sigma_{\text {ess }}\left(\mathcal{L}_{\star}\right)=\sigma_{\text {ess }}\left(\mathcal{L}_{\infty}\right)=(-\infty,-1],
$$

which shows that $\mathcal{L}_{\star}$ is Fredholm with index 0 .

Next, recall from Lemma 4.4 that the kernel of $\mathcal{L}_{\star}$ consists of bounded functions $\left(a_{0}, b_{0}\right)$ which solve the system (4.10), and that these solutions have the asymptotic behavior in (4.11), as $x \rightarrow \infty$, and (4.12), as $x \rightarrow-\infty$. Also, recall that $\left(A_{0}^{\star \prime}, B_{0}^{\star \prime}\right)$ belongs to the kernel of $\mathcal{L}_{\star}$, which is therefore at least one-dimensional, and that

$$
A_{0}^{\star \prime}(x)=-\alpha_{\star} e^{-x}+\mathcal{O}\left(e^{-(1+\delta) x}\right), \quad B_{0}^{\star \prime}(x)=\beta_{\star} e^{-\sqrt{2} x}+\mathcal{O}\left(e^{-(\sqrt{2}+\delta) x}\right), \quad \alpha_{\star}>0, \quad \beta_{\star}>0,
$$

as $x \rightarrow \infty$, for some $\delta>0$.

Suppose that the kernel of $\mathcal{L}_{\star}$ were not one-dimensional. Then there exists a solution $\left(a_{0}, b_{0}\right)$ of $(4.10)$ such that $\left(a_{0}, b_{0}\right)$ and $\left(A_{0}^{\star \prime}, B_{0}^{\star \prime}\right)$ are linearly independent. We can also assume that $b_{0}(x)>0$ for sufficiently large $x$. According to Lemma 4.4, $a_{0}$ and $b_{0}$ have the asymptotic behavior

$$
a_{0}(x)=\alpha_{+} e^{-x}+\mathcal{O}\left(e^{-(1+\delta) x}\right), \quad b_{0}(x)=\beta_{+} e^{-\sqrt{2} x}+\mathcal{O}\left(e^{-(\sqrt{2}+\delta) x}\right), \quad \alpha_{+}<0, \quad \beta_{+}>0,
$$


as $x \rightarrow \infty$, for some $\delta>0$. Now the linear combination $\alpha_{+}\left(A_{0}^{\star \prime}, B_{0}^{\star \prime}\right)+\alpha_{\star}\left(a_{0}, b_{0}\right)$ belongs to the kernel of $\mathcal{L}_{\star}$, and does not vanish identically. In particular $\alpha_{+} B_{0}^{\star \prime}+\alpha_{\star} B_{0}$ is either negative or positive for large $x$, and

$$
\left(\alpha_{+} A_{0}^{\star \prime}+\alpha_{\star} a_{0}\right)(x)=\mathcal{O}\left(e^{-(1+\delta) x}\right) .
$$

This contradicts the result in Lemma 4.4, and implies that the kernel of $\mathcal{L}_{\star}$ is one-dimensional.

Finally, we show that the generalized kernel of $\mathcal{L}_{\star}$ is also one-dimensional. Assuming that $\left(a_{0}, b_{0}\right)$ is a principal vector in the generalized kernel of $\mathcal{L}_{\star}$, we have that

$$
\begin{aligned}
& a_{0}^{\prime \prime}+\left(1-3 A_{0}^{\star 2}-2 B_{0}^{\star 2}\right) a_{0}-4 A_{0}^{\star} B_{0}^{\star} b_{0}=A_{0}^{\star \prime} \\
& b_{0}^{\prime \prime}+\left(1-2 A_{0}^{\star 2}-3 B_{0}^{\star 2}\right) b_{0}-4 A_{0}^{\star} B_{0}^{\star} a_{0}=B_{0}^{\star \prime} .
\end{aligned}
$$

Multiplying the first equation by $A_{0}^{\star \prime}$, the second one by $B_{0}^{\star \prime}$, integrating over $\mathbb{R}$, and taking the sum of the resulting equalities we find the contradiction

$$
\begin{aligned}
\int_{\mathbb{R}}\left(A_{0}^{\star \prime}\right)^{2}+\left(B_{0}^{\star \prime}\right)^{2} d x= & \int_{\mathbb{R}} a_{0}\left(A_{0}^{\star \prime \prime \prime \prime}+\left(1-3 A_{0}^{\star 2}-2 B_{0}^{\star 2}\right) A_{0}^{\star \prime}-4 A_{0}^{\star} B_{0}^{\star} B_{0}^{\star \prime}\right) d x \\
& +\int_{\mathbb{R}} b_{0}\left(B_{0}^{\star \prime \prime \prime \prime}+\left(1-2 A_{0}^{\star 2}-3 B_{0}^{\star 2}\right) B_{0}^{\star \prime}-4 A_{0}^{\star} B_{0}^{\star} A_{0}^{\star \prime}\right) d x=0 .
\end{aligned}
$$

\subsection{Persistence of heteroclinic orbits}

Solving the system (4.2) is equivalent to solving the equation

$$
\mathcal{T}\left(A_{0}, B_{0}, \bar{c}, \bar{\mu}, \mu_{*}\right)=0,
$$

where

$$
\mathcal{T}\left(A_{0}, B_{0}, \bar{c}, \bar{\mu}, \mu_{*}\right)=\left(\begin{array}{c}
A_{0}^{\prime \prime}+A_{0}-A_{0}\left(A_{0}^{2}+2 B_{0}^{2}\right)+\bar{c} A_{0}^{\prime}-\mathcal{R}_{A}^{*}\left(A_{0}, A_{0}^{\prime}, B_{0}, B_{0}^{\prime}, \bar{\mu}, \bar{c} ; \mu_{*}\right) \\
B_{0}^{\prime \prime}+B_{0}-B_{0}\left(2 A_{0}^{2}+B_{0}^{2}\right)+\bar{c} B_{0}^{\prime}-\mathcal{R}_{B}^{*}\left(A_{0}, A_{0}^{\prime}, B_{0}, B_{0}^{\prime}, \bar{\mu}, \bar{c} ; \mu_{*}\right)
\end{array}\right)
$$

A particular solution of $(4.14)$ is $\left(A_{0}^{\star}, B_{0}^{\star}, 0,0,0\right)$, with linearization

$$
D_{\left(A_{0}, B_{0}\right)} \mathcal{T}\left(A_{0}^{\star}, B_{0}^{\star}, 0,0,0\right)=\mathcal{L}_{\star}, \quad \partial_{\bar{c}} \mathcal{T}\left(A_{0}^{\star}, B_{0}^{\star}, 0,0,0\right)=\left(A_{0}^{\star \prime}, B_{0}^{\star \prime}\right) .
$$

Recall that the linear operator $\mathcal{L}_{\star}$ is Fredholm with index 0 , and that 0 is an algebraically simple eigenvalue of $\mathcal{L}_{\star}$, with associated eigenvector $\left(A_{0}^{\star \prime}, B_{0}^{\star \prime}\right)$. Consequently, $\left(A_{0}^{\star \prime}, B_{0}^{\star \prime}\right)$ does not belong to the range of $\mathcal{L}_{\star}$ and we conclude that the differential $D_{\left(A_{0}, B_{0}, \bar{c}\right)} \mathcal{T}\left(A_{0}^{\star}, B_{0}^{\star}, 0,0,0\right)$ is onto. This allows to solve the equation (4.14) for $\left(A_{0}, B_{0}\right)=\left(A_{0}, B_{0}\right)\left(\bar{\mu}, \mu_{*}\right)$ and $\bar{c}=\bar{c}\left(\bar{\mu}, \mu_{*}\right)$, in a neighborhood of the particular solution $\left(A_{0}^{\star}, B_{0}^{\star}, 0,0,0\right)$. The projection of the leading-order perturbation term, $\left(\left(\beta-2 \sqrt{\mu_{*}}\right)^{-1} A_{0}^{\prime \prime},\left(\beta+2 \sqrt{\mu_{*}}\right)^{-1} B_{0}^{\prime \prime}\right)$ along the kernel actually vanishes, so that at leading order the speed vanishes. This proves the following result

Lemma 4.7 (Existence of heteroclinic orbits) For any $\bar{\mu}>0$ and $\mu_{*}>0$ sufficiently small, there exists a speed $\bar{c}=\bar{c}\left(\bar{\mu}, \mu_{*}\right)=\mathcal{O}\left(\mu_{*}^{1 / 2}+\bar{\mu}^{1 / 2}\right)$, such that the system (4.2) possesses a heteroclinic orbit

$$
\left(A_{0}, B_{0}\right)=\left(A_{0}, B_{0}\right)\left(\bar{\mu}, \mu_{*}\right)=\left(A_{0}^{\star}, B_{0}^{\star}\right)+\mathcal{O}\left(\mu_{*}^{1 / 2}+\bar{\mu}^{1 / 2}\right) .
$$

Going backwards through the different scalings and the reduction procedure, we obtain our main result, Theorem 1, which shows the existence of dislocations in the modified Swift-Hohenberg equation (1.1). 


\section{Discussion and numerical computations}

\subsection{Summary and generalizations}

We showed existence of dislocations. Our model was an anisotropic Swift-Hohenberg equation that allows for rolls with orientation close to a fixed wave vector, only. The proof finds dislocations as interfaces between roll solutions with rationally dependent wavenumbers. Such roll solutions bifurcate from the trivial state when suitable periodic boundary conditions are imposed in the direction of the wave vector. The assumption of anisotropy guarantees that, close to onset, only two roll solutions bifurcate. The traveling-wave equation for interfaces between the rolls is a coupled system of secondorder complex equations for the amplitude of the two different resonant modes. The two key steps in our analysis are to show that

- one can rigorously separate the amplitudes of two resonant wave vectors from the many other modes that bifurcate for nearby parameter values;

- the heteroclinic orbits in the traveling-wave equation that describe dislocations are robust.

The rigorous separation of modes is achieved using a center-manifold reduction for a spatial dynamics formulation together with appropriate scalings before and after reduction. Heteroclinic orbits for the leading-order reduced model were found in [12] using variational methods. We refine their results slightly and show, in particular, robustness of the heteroclinics, using comparison techniques. Robustness can be achieved only for even solutions. The leading-order phase-averaging symmetry gives in fact a continuum of heteroclinic orbits, obtained by shifting one of the modes relative to the other. We expect that most of those solutions disappear when higher-order terms in the expansion are taken into account. On the other hand, one expects that at least one such non-symmetric heteroclinic persists for the full system. In fact, a Melnikov analysis shows that one can reduce the persistence problem to finding zeros of a function $M(\varphi)$, where $\varphi \in S^{1}$ represents the shift of one of the roll patterns. The function $M(\varphi)$ vanishes at $\varphi=0$ and $\varphi=\pi$ due to reflection symmetries. Both zeros are in fact conjugate by the symmetry $u \mapsto-u$, so that $M(\varphi)=M(\varphi+\pi)=M(-\varphi)$. When higher-order terms are taken into account, this implies that there exists at least one root in addition to the dislocations that we found, here. One can think of these additional dislocations as saddle points at the boundaries of the basin of attractions of dislocations with $\varphi=0$ and $\varphi=\pi$. In fact, we expect that the sign of $M^{\prime}$ at roots actually gives a stability index, so that our analysis predicts at least one stable and one unstable dislocation up to symmetries.

The reduction method presented here is quite robust and should apply to a number of more general set-ups. First of all, we expect that the analysis goes through, with appropriate changes in the scalings, when quadratic terms are present in the nonlinearity,

$$
u_{t}=-(1+\Delta)^{2} u+\mu u+\beta \partial_{x}^{2} u+c_{1} u^{2}+c_{2}|\nabla u|^{2}-u^{3},
$$

provided that the bifurcation of roll solutions is supercritical. The reduced equations are essentially unchanged, up to a factor for the nonlinearity. In particular, the leading-order reflection symmetry $A_{0} \leftrightarrow B_{0}$ is preserved, and the mixed terms $A_{0}\left|B_{0}\right|^{2}$ carry a factor two relative to single-mode terms $A_{0}\left|A_{0}\right|^{2}$ in (4.3). This can be verified by a somewhat lengthy calculation (note that it is sufficient to 
consider the steady-state problem $\partial_{x}=0$ ) or using the somewhat formal amplitude expansion presented below. We believe that the analysis can be generalized to pattern-forming systems such as anisotropic reaction-diffusion or Rayleigh-Bénard convection.

\subsection{Amplitude equations}

An alternate approach to the existence problem would start with the derivation of a modulation equation. Scaling $x=\varepsilon \tilde{x}, y=\varepsilon \tilde{y}, t=\varepsilon^{2} \tilde{t}$, and substituting an Ansatz $u(t, x, y)=U(\tilde{t}, \tilde{x}, \tilde{y}) \mathrm{e}^{\mathrm{i} y}+c . c$. , with $\varepsilon^{2}=\mu$, one obtains at leading order the amplitude equation,

$$
U_{\tilde{t}}=\beta U_{\tilde{x} \tilde{x}}+4 U_{\tilde{y} \tilde{y}}+U-3 U|U|^{2} .
$$

The traveling-wave equation possesses invariant subspaces spanned by solutions of the form $U_{m} \mathrm{e}^{\mathrm{i} m y}$. However, sums of such subspaces are in general not flow-invariant. One therefore needs to perform a similar reduction to a center-manifold for this amplitude equation in order to find dislocations which connect solutions with different wavenumber $k$. Note that the $y$-periodic boundary conditions that we imposed in our proof need to be replaced by twisted boundary conditions $U(x, 0)=\mathrm{e}^{\mathrm{i} \psi} U(x, L)$ for some appropriate $\psi$.

Both methods, direct reduction of the traveling-wave equation, or derivation of amplitude equations followed by ODE reduction for the traveling-wave problem, yield the same reduced system of equations. This can be seen, for instance, by either invoking an approximation result for amplitude equations that guarantees correct approximation to a given order in $\varepsilon$, or by comparing the algebra in the reduction process. By first deriving the amplitude equation, it is however somewhat easier to see that the reduced equations are unchanged at leading order when quadratic terms are present in the original problem. To see this, note that the amplitude equation does not change except for the coefficient of the cubic term, since quadratic terms average out. The ansatz $U=A \mathrm{e}^{\mathrm{i} k_{-} y}+B \mathrm{e}^{\mathrm{i} k_{+} y}+$ c.c. into the nonlinearity then gives the particular form $A\left(|A|^{2}+2|B|^{2}\right)$ by simply expanding the cubic.

\subsection{Free energy and Peach-Köhler force}

The anisotropic Swift-Hohenberg equation is an $L^{2}$-gradient flow with respect to the free energy

$$
E(u)=\int_{\mathbb{R}^{2}}\left(\frac{1}{2}((1+\Delta) u)^{2}+\frac{\beta}{2}\left(\partial_{x} u\right)^{2}-\frac{\mu}{2} u^{2}+\frac{1}{4} u^{4}\right) \mathrm{d} x \mathrm{~d} y .
$$

Of course, the gradient flow has to be understood in a formal sense since the energy is infinite for almost all interesting solutions.

The variational structure implies that the reduced equations on our center-manifold are in fact Hamiltonian with respect to a reduced symplectic structure. In particular, stationary solutions can be found from a least-action principle. Traveling-wave equations possess a Lyapunov-function, which allows for topological methods in the construction of connecting orbits.

We emphasize that our methods do not rely on any of these considerations. The reduced equation turns out to be Hamiltonian even when the original problem does not possess a free energy, for instance when terms of the form $|\nabla u|^{2}$ are included. Traveling waves are obtained from a simple transversality argument. 
On the other hand, one can compare our results with simple predictions from the variational structure when a free energy is present. A rough argument would predict that the speed of the dislocation is proportional to the difference in energies between the different rolls. Indeed, rolls $u=u_{k}(k y)$ with different wavenumbers typically possess different energies per unit volume,

$$
E_{k}(u)=f\left(\frac{1}{2}\left(\left(1+\partial_{y y}\right) u_{k}\right)^{2}-\frac{\mu}{2} u_{k}^{2}+\frac{1}{4} u_{k}^{4}\right) \mathrm{d} y
$$

The difference $E_{k_{+}}(u)-E_{k_{-}}(u)$ creates an effective force, the Peach-Koehler force [8], that drives the motion of the dislocation in the $x$-direction.

In our situation, one can expand the energy difference in $\mu_{*}$ and $\bar{\mu}$. At leading order, $E_{k}(u)=-\bar{\mu}^{2} / 6$ does not depend on $k$. This is in agreement with our analysis, where at leading order, the speed vanishes.

Furthermore, one can consider the wavenumbers $k=k_{*}$ as an independent parameter and carry out the reduction construction. The wavenumber appears at leading order as a shift in the onset of instability, factoring the linear terms $A_{0}$ and $B_{0}$ in the equations for $A_{0}^{\prime}$ and $B_{0}^{\prime}$, respectively. The resulting Melnikov integral does not vanish since for example $\int A_{0} A_{0}^{\prime}=A_{0}^{2}(+\infty)-A_{0}^{2}(-\infty) \neq 0$. Similarly, the change in wavenumber leads to a shift in energy $E_{k}$, which again confirms the prediction of a force being proportional to the energy difference at leading order.

\subsection{Numerical computations}

We simulated the isotropic and anisotropic Swift-Hohenberg equation in a square of length 20 with periodic boundary conditions using a spectral code with $256 \times 256$ Fourier modes in space and implicit 2nd order time stepping. We used $\mu=0.3$ and $\beta=0$ and $\beta=2$, respectively. Initial conditions were chosen as $\cos \left(2 \pi \ell_{ \pm} y\right)$ for $\pm x>10$, smoothly interpolated on $|x|<10$. In all examples, $\left|\ell_{+}-\ell_{-}\right|=1$. Higher differences simply produce multiple dislocations. We chose $\ell_{+}=20$ and $\ell_{-}=19$ in one set of examples, and $\ell_{+}=21$ and $\ell_{-}=20$ in the other. The wavenumber $\ell=20$ is the linearly preferred wavenumber. In all cases, we observe the predicted drift of the dislocations in the direction of the preferred wavenumber; see Figure 5.1. The drift generally is faster in the anisotropic case. Also, dislocations generally have stronger decay in the direction transverse to the rolls in the presence of anisotropy. In the isotropic case, the rolls with wavenumber smaller than the critical wavenumber are laterally unstable, the so-called zig-zag instability. It appears that the dislocations are unstable in this case, too. In the anisotropic case, this instability is suppressed. In general, however, we found little qualitative differences between the two cases $\beta=0$ and $\beta=2$. We also computed the heteroclinics in the bifurcation equation using continuation software AUTO07P. We therefore used a linear homotopy from (4.3) to the trivial equation

$$
\begin{aligned}
& A_{+}^{\prime \prime}=A_{+}-1, \\
& A_{-}^{\prime \prime}=-A_{-}\left(1-A_{-}^{2}\right),
\end{aligned}
$$

where $A_{ \pm}=A_{0} \pm B_{0}$, and trivial heteroclinic $A_{-}(x)=\tanh (x / \sqrt{2}), A_{+}=1$. We plotted the resulting functions $A_{0}, B_{0}$ in Figure 5.2. From $A_{0}, B_{0}$, we can predict the shape of dislocations at leading order according to Theorem 1. We plotted level sets of this leading-order approximation in Figure 5.2. We chose parameters $\sqrt{\bar{\mu} / \beta}=\sqrt{0.3} / 2, \ell_{\star}=20$ roughly corresponding to the values in Figure 5.1. 

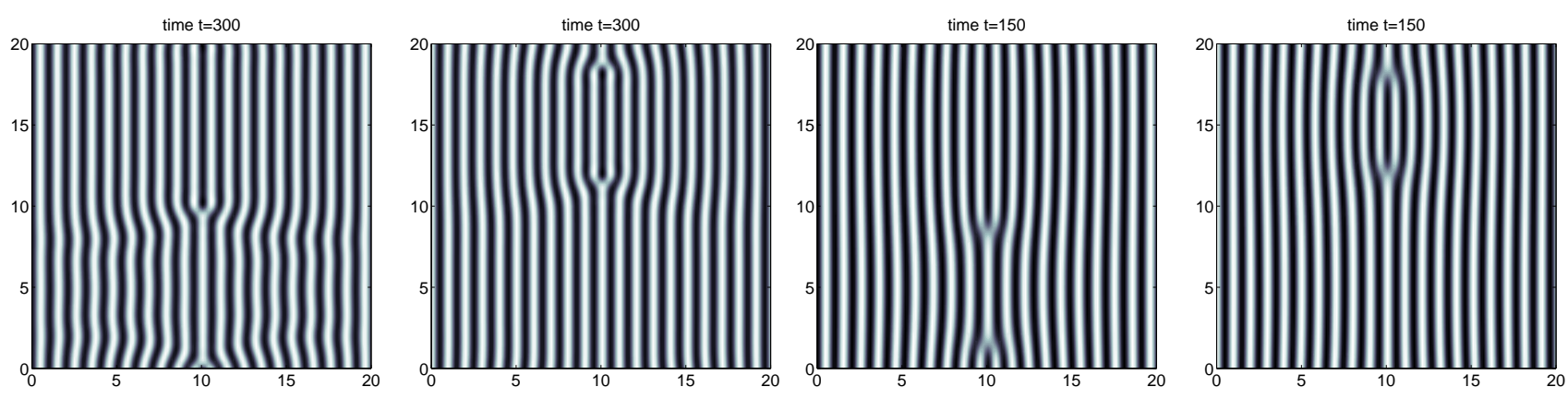

Figure 5.1: Direct simulation of the isotropic (left two pictures) and anisotropic Swift-Hohenberg equation. In each case, the left picture corresponds to $\ell_{+}=21, \ell_{-}=20$, and the right picture to $\ell_{+}=20, \ell_{-}=19$. Note that time elapsed is double in the isotropic case.
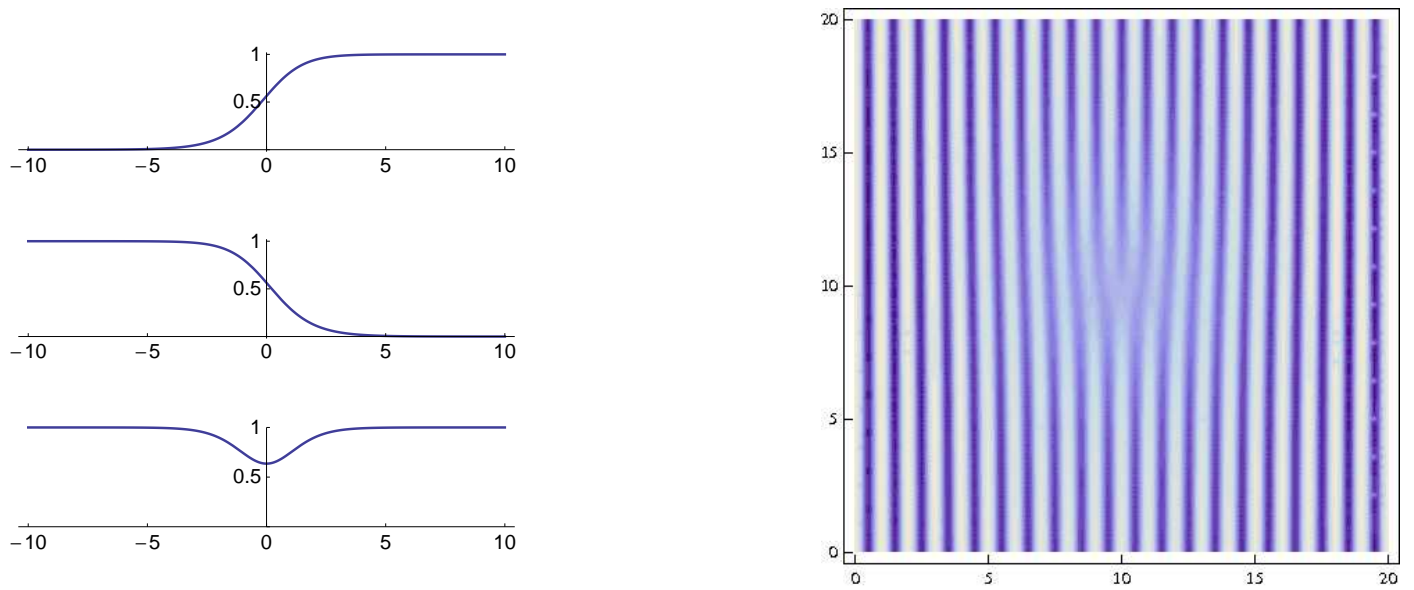

Figure 5.2: On the left, plot of $A_{0}, B_{0}$, and $A_{0}^{2}+B_{0}^{2}$, from top to bottom, computed for (4.3). The right-hand picture shows the leading-order approximation to the Swift-Hohenberg equation: $u(x, y)$ as defined in (1.3), Theorem 1.

We emphasize that our analysis is valid only in a very small neighborhood of the origin. In the present example, $\ell_{\star}=20$ gives $\mu_{\star}=2.5 \cdot 10^{-3}$, and $\bar{\mu} \ll \mu_{\star}^{2} \sim 6.25 \cdot 10^{-6}$. One would therefore not expect quantitative agreement between the results of Figure 5.1 and 5.2. Nevertheless, we were still quite surprised to find the apparent agreement between Figures 5.1 and 5.2, which may well reflect a common observation that local bifurcation analysis gives good predictions far beyond its domain of rigorous validity.

\section{References}

[1] D. Boyer. Numerical study of domain coarsening in anisotropic stripe patterns. Phys. Rev. E 69 (2004), 066111.

[2] M. Cross and P. Hohenberg. Pattern formation outside of equilibrium. Rev. Mod. Phys. 65 (1993), 851-1112. 
[3] N. Ercolani, R. Indik, A. Newell, and T. Passot. The geometry of the phase diffusion equation. J. Nonlinear Sci. 10 (2000), 223-274.

[4] N. Ercolani, R. Indik, A. Newell, and T. Passot. Global description of patterns far from onset: a case study. Complexity and nonlinearity in physical systems (Tucson, AZ, 2001). Phys. D 184 (2003), 127-140.

[5] N. Ercolani and S. Venkataramani. A variational theory for point defects in patterns. J. Nonlinear Sci. 19 (2009), 267-300.

[6] M. Haragus and G. Iooss. Local bifurcations, center manifolds, and normal forms in infinite dimensional dynamical systems. Universitext. Springer-Verlag London, Ltd., London; EDP Sciences, Les Ulis, 2011. 329 pp.

[7] C. Kamaga, F. Ibrahim, and M. Dennin. Dislocation dynamics in an anisotropic stripe pattern. Phys. Rev. E 69 (2004), 066213.

[8] M. Peach and J. S. Koehler. The forces exerted on dislocations and the stress fields produced by them. Phys. Rev. 80 (1950), 436-439.

[9] L. Pismen. Patterns and interfaces in dissipative dynamics. With a foreword by Y. Pomeau. Springer Series in Synergetics. Springer-Verlag, Berlin, 2006.

[10] H. Qian and G. Mazenko. Growth of order in an anisotropic Swift-Hohenberg model. Phys. Rev. E 73 (2006), 036117.

[11] A. Roxin and H. Riecke. Rotating convection in an anisotropic system. Phys. Rev. E 65, 046219.

[12] G. van den Berg, R. van der Vorst. A domain-wall between single-mode and bimodal states. Diff. Int. Equations 13 (2000), 369-400. 